\title{
Recent Advances in Theoretical and Physical Aspects of NMR Chemical Shifts
}

\author{
Angel C. De Dios ${ }^{a^{*}}$, and Cynthia Juan Jameson ${ }^{b}$ \\ ${ }^{a}$ Department of Chemistry, Georgetown University, $37^{\text {th }}$ and O Streets, NW, Washington, D. C., USA \\ ${ }^{b}$ Department of Chemistry, University of Illinois at Chicago, $845 \mathrm{~W}$. Taylor St., Chicago, Illinois, US A
}

In the first part of this review, theoretical aspects of nuclear magnetic shielding include (a) general theory, for example, newly developed approaches in relativistic theory of nuclear shielding, the relation between the spin-rotation tensor and shielding in relativistic theory, ab initio methods for treating open shell systems and a complete theory of chemical shifts in paramagnetic systems, the link between the definitions of the elusive concepts aromaticity and anti-aromaticity and the magnetic properties: the magnetizability tensor and the nuclear magnetic shielding tensor via delocalized electron currents and electron current maps, (b) ab initio and DFT calculations, both relativistic and non-relativistic, for various nuclei in various molecular systems using various levels of theoretical treatment. Physical aspects include (a) anisotropy of the shielding tensor, usually from solid state measurements, and calculations to support these, (b) shielding surfaces and rovibrational averaging, paying special attention to the sensitive relationship between shielding and bond angles or torsion angles that makes shielding such a powerful tool for structural/conformational determination in macromolecules, (c) chemical shifts that arise from isotopic substitution of NMR nucleus or neighboring nuclei, (d) intermolecular effects on nuclear shielding, and (e) absolute shielding scales.

Keywords: nuclear magnetic shielding; ab initio; relativistic; intermolecular effects; tensor; isotope shift

\section{THEORETICAL ASPECTS OF NUCLEAR SHIELDING}

Chemical shifts that are observed in nuclear magnetic resonance spectroscopy are related to the difference between the fundamental quantities, the nuclear magnetic shielding, of the nucleus in the sample and the nucleus in the reference substance. Theoretical calculations focus on the fundamental quantity, the nuclear magnetic shielding, $\sigma$ relative to the bare nucleus, rather than the shielding differences obtained from experiment, that are based on a choice of reference substance.

$$
\text { chemical shift, } \delta \equiv\left(v-v_{\text {ref }}\right) / v \text { ref }=\left[\sigma_{\text {ref }}-\sigma_{\text {sample }}\right] /\left[1-\sigma_{\text {ref }}\right](\text { Equation } 1)
$$

Here all quantities are tensors, in general, so the chemical shift tensor components $\delta_{\mathrm{xx}}$ can be related to shielding tensor components $\sigma_{\mathrm{xx}}$. Shielding is essentially a response of electrons 
to the presence of a magnetic field. Except for highly symmetric molecules, the observed shielding is expected to be dependent on the orientation of a molecule with respect to the magnet. However, due to the rapid and random tumbling of molecules in the liquid or gas phase, only the isotropic value of shielding, the average value, is observed. In the solid state where rapid tumbling does not occur, the orientation dependence or the anisotropy of the shielding manifests. The measurement of shielding as a tensor quantity obviously provides much more information than the isotropic value alone regarding the structure and dynamics of a molecule. Ab initio calculations of course yield the full shielding tensor. Thus, progress in understanding nuclear shielding, both its theoretical and physical aspects, has been dependent on measurements in the solid state.

The symmetric part of the shielding tensor is characterized by 6 numbers (components) for the nuclear site that has the lowest symmetry, some of these 6 numbers are either zero or are identical, depending on the symmetry at the nuclear site. The shielding tensor can be characterized by specifying the values of the principal components and the orientation of the principal axis system, again, in general requiring 6 numbers. The numbering of the principal components is such that $\sigma_{33} \geq \sigma_{22} \geq$ $\sigma_{11}$, that is, the least shielded tensor component is called $\sigma_{11}$ and the most shielded component $\sigma_{33}$.

The shielding tensor is also characterized by combinations of these numbers, the span and the skew. The span, $\Omega$, always a positive quantity, is the difference between the most shielded $\left(\sigma_{33}\right)$ and the least shielded tensor component $\left(\sigma_{11}\right)$, corresponding to the width of the resonance powder pattern in ppm. That is, where $\sigma_{33} \geq \sigma_{22} \geq \sigma_{11}$

$$
\Omega \equiv\left(\sigma_{33}-\sigma_{11}\right)
$$

and the skew $\kappa$ is a signed dimensionless quantity, defined as

$$
\kappa \equiv 3\left(\sigma_{\text {iso }}-\sigma_{22}\right) /\left(\sigma_{33}-\sigma_{11}\right)
$$

The skew ranges from -1 (for $\sigma_{22}=\sigma_{33}$ ) to +1 (for $\sigma_{11}=\sigma_{22}$ ). Experimentally, the skew is independent of the reference substance used in defining the chemical shift,

$$
\kappa \equiv 3\left(\delta_{\text {iso }}-\delta_{22}\right) /\left(\delta_{33}-\delta_{11}\right)
$$

but the span cannot be determined exactly purely from experiment because

$$
\begin{aligned}
\Omega \equiv & \left(\sigma_{33}-\sigma_{11}\right) \\
& =\left(\delta_{11}-\delta_{33}\right) /\left(1-\sigma_{\text {ref }}\right)
\end{aligned}
$$

and $\sigma_{\text {ref }}$ is not always negligible compared to 1 , considering the level of accuracy that chemical shifts can be measured nowadays. The $\left(1-\sigma_{\text {ref }}\right)$ factor is usually incorrectly left out in converting chemical shifts from one reference to another (which should not happen if one thinks always in terms of the fundamental molecular electronic property, shielding, from which chemical shifts are derived). We should note the $\left(1-\sigma_{\text {ref }}\right)$ factor here and always be conscious of our inability to know span exactly from measuring the width of the resonance powder pattern $\left(\delta_{11}-\delta_{33}\right)$.

General Theory. As an introduction to some of the ideas and terms used in this review, it would benefit the reader to first look at the lucid and accessible introduction to relativistic effects by Jochen Autschbach in a Perspective, ending with special attention to NMR quantities: electric field gradients, chemical shifts and J coupling (Autschbach, 2012). The reason that relativistic effects are so important for these NMR quantities is that $\mathrm{r}^{-3}$ appears in the mathematical operators for calculations of these quantities, heavily weighting the electron contributions very close to the nucleus where consequences of Einstein's special relativity in quantum chemistry, the relativistic effects, are large. For low atomic numbers, we can still approximately use non-relativistic theory but many NMR experiments use nuclei below the first and second row of the Periodic Table, where non-relativistic theory becomes inadequate. 
Wenjian Liu continues to re-cast and explain the basic ideas of relativistic quantum chemistry at various levels of exposition: comprehensive advances (Liu, Advances, 2014; Liu, Perspectives, 2014), and tutorial (Liu, 2015), (these are only the most recent ones), so as to answer these fundamental questions: (a) what is the appropriate relativistic many-electron Hamiltonian, (b) how to make explicit representation of relativistic wavefunctions, (c) how to formulate relativistic molecular electronic properties, and (d) how to interface relativistic quantum chemistry and quantum electrodynamics. These fundamental issues are to be addressed from both conceptual and methodological standpoints, so as to establish the 'big picture' of relativistic molecular quantum mechanics. In particular, a more general formulation of the so-called exact two-component (X2C) relativistic Hamiltonians is given where various kinetic and magnetic balances are incorporated in a unified manner (Liu, Advances, 2014). In a manner of speaking, Liu comments that $\mathrm{X} 2 \mathrm{C}$ serves as a seamless bridge between the Schrödinger (nonrelativistic) and Dirac (relativistic) equations. In these expositions, special mention is made of electric and magnetic properties, nuclear magnetic shielding, in particular.

For systems where spin-orbit couplings are very weak, it is highly desired to separate the algebraic exact two-component (X2C) relativistic Hamiltonian into spin-free and spin-dependent terms, such that scalar relativistic effects can be treated variationally to infinite order, whereas spin-orbit couplings can be treated order by order either perturbatively or variationally (Li et al., 2012). This is done by a priori partitioning the Dirac matrix into spin-free and spin-dependent terms. Recently, this idea is extended to both electric and magnetic molecular properties $(\mathrm{Li}$ et al., 2014). Explicit expressions are derived for these properties as well as two-electron spin-orbit couplings. The spin-dependent terms are treated via analytic derivative technique. Whereas here the spin-orbit coupling can be treated order by order, as needed, in an earlier version, the full X2C-
NMR formulation of nuclear magnetic shielding, the mixed second-order energy is of first order in both the external magnetic field $\mathbf{B}_{0}$ and the nuclear moment $\boldsymbol{\mu}_{\mathrm{N}}$ but is of infinite order in spin-orbit coupling (Sun et al., 2012). This is one extreme. At the other extreme, sf-X2C-NMR is zeroth order in spinorbit coupling (Cheng et al., 2013). Both the full X2C (Jameson and de Dios, 2014) and the sfX2C formulations (Jameson and de Dios, 2015) have been reviewed by us earlier.

The well-known nonrelativistic mapping of the nuclear magnetic shielding tensor and the nuclear spin-rotation coupling tensor proposed by Ramsey in 1950 and made generally useful by Flygare since 1964, had been in wide use for establishing absolute shielding scales for light nuclei in molecules containing light atoms (Ramsey, 1950; Flygare, 1964). The direct relativistic mapping between nuclear magnetic shielding tensor and the nuclear spin-rotation coupling tensor (Xiao et al., 2012) [reviewed by us earlier (Jameson and de Dios, 2013)] permits accurate determination of absolute shielding scales for nuclei in relativistic systems. What is new is the employment of rotational London orbitals in the basis set for the 4-component relativistic calculations of the nuclear spinrotation coupling tensor (Xiao et al., 2014). These orbitals are the counterpart of GIAO (gauge-including atomic orbitals) in nuclear magnetic shielding calculations. This is to help overcome the slow convergence with basis set size in relativistic calculations of spin-rotation tensors. It is well known that the advantages of using London orbitals in NMR shielding calculations are that (a) the unphysical dependence on the gauge origin can be eliminated completely, (b) the slow basis set convergence problem is greatly alleviated. At first glance the nuclear spin-rotation tensor does not have a gauge origin problem since the tensor is defined physically relative to the position of the nucleus in question, but the transformation of the origin of the electronic orbital angular momentum to an arbitrary origin holds only in the limit of a complete basis set and therefore leads to an origin dependence with a finite basis set. Thus, the 
use of London orbitals endows the same twofold advantage here as use of GIAOs does to NMR shielding. The use of kinetically balanced rotational London orbitals (KBRLO) ensures that the relativistic treatment gives rise to the correct nonrelativistic limit, even with a finite basis set. Also, the use of KB-RLO and GIAO together gives rise to a direct relativistic mapping between nuclear magnetic shielding and nuclear spin-rotation coupling tensors that is identical to the one derived previously without these pre-factors in the basis set, as it has to be, because the mapping is insensitive to one-particle basis sets, as shown previously (Xiao et al., 2012).

\section{Relativistic formulations with various} approximations have recently been reported (Reynolds and Shiozaki, 2015; Yoshizawa and Hada, 2015). One is a non-perturbative implementation of the 4-component DiracHartree-Fock (DHF) method for molecules in magnetic fields using the Dirac-CoulombGaunt Hamiltonian (Reynolds and Shiozaki, 2015). The DHF wavefunction is constructed from GIAOs; restricted magnetic balance is used to generate 2-spinor basis functions for the small component. The molecular integrals for the Coulomb and Gaunt interactions are computed using density fitting. Timings for an 85- and a 112-atom molecule are given. The other is a second-order Douglas-Kroll-Hess method where the magnetic operators are replaced with the Breit-Pauli magnetic operators and GIAOs are used (Yoshizawa and Hada, 2015). Shielding tensors for $\mathrm{HXeCCH}$ molecule are reported as an example, in addition to diatomic molecules containing iodine, $\mathrm{IX}(\mathrm{X}=\mathrm{F}, \mathrm{Cl}, \mathrm{Br})$.

In a holistic review article, Lazzeretti shows that the non-relativistic quantum mechanical theory of magnetic properties can be proposed in terms of electronic current densities induced by an external magnetic field and permanent magnetic dipole moments at the nuclei. The molecular response to magnetic perturbations is reformulated in terms of electron current density induced by magnetic fields and nuclear magnetic dipoles. Theoretical relationships are derived for the evaluation of magnetizability, nuclear magnetic shielding, and nuclear spin-spin coupling, proving that the whole theory can be reformulated via the equations of classical electromagnetism, provided only that the current density is evaluated by quantum mechanical methods. Emphasis is placed on the invariance of response properties in a translation of the coordinate system as a basic requirement for measurability. In principle, only origin-independent atomic and molecular macroscopic observables can be experimentally accessible: A measurement is not generally conceivable for quantities which change in a translation of coordinate system. For the same reason, translationally invariant theoretical definitions are needed for a meaningful evaluation of response properties via computational procedures of quantum mechanics. In this review, the connections among translational invariance, gauge invariance, and electron charge conservation are outlined, showing that they can be illustrated via quantum mechanical sum rules, in typical Lazzeretti fashion (Lazzeretti, 2014). He employs the Bloch gauge to define electric and magnetic multipole electron operators. Including the terms in cubic response to magnetic field $\mathbf{B}$, he also derives the expressions for hypershielding (the quadratic term in the $\mathbf{B}$ dependence of nuclear magnetic shielding) and for hypermagnetizability. The sum rules are not necessarily obeyed in calculations using the algebraic approximation, that is, within a finite basis set, but the closeness to following the sum rules is a measure of basis set completeness.

The electron density of atoms is set in rotation by an external magnetic field and thus, by continuity of the flow, peripheral delocalized electron currents are expected for any molecule. The interest in delocalized electron currents and electron current maps is intimately linked with definitions of the elusive concepts of aromaticity and antiaromaticity through the magnetic properties: the magnetizability tensor and the nuclear magnetic shielding tensor. It had been assumed that delocalized currents require a ring of chemically bonded atoms to sustain 
them. Pelloni et al. show that delocalized currents are induced by magnetic fields without a ring of bonded atoms or cyclic system (Pelloni et al., 2014). Fairly large delocalized electron flow is calculated for any molecules containing at least three atoms, such as $\mathrm{H}_{2} \mathrm{O}$ or $\mathrm{NH}_{3}$. They conclude that the ability to sustain delocalized currents is not exclusive to molecules characterized by a ring structure. They have documented electron motion all over an interatomic circuit, induced by a magnetic field in a series of neutral or charged molecules via the quantum mechanical current density. For example, for $\mathrm{H}_{2} \mathrm{O}$ in two different orientations, the total electron stream is comparable to that in benzene molecule $(116 \%$ and $105 \%$ of the benzene value). The delocalized currents are less intense in species such as $\mathrm{CO}_{3}{ }^{2-}$ and $\mathrm{NO}_{3}{ }^{-}$ because of electron withdrawing exerted by electronegative peripheral atoms. This work brings into question the use of $\mathrm{NICS}_{||}$(the parallel component of the NICS calculated at the center of the benzene ring) for the molecule as a reliable quantifier of absolute aromaticity based on the magnetic criterion. On this basis one would call $\mathrm{H}_{2} \mathrm{O}, \mathrm{NH}_{3}, \mathrm{CH}_{4}$, $\mathrm{H}_{3} \mathrm{O}^{+}, \mathrm{NH}_{4}{ }^{+}$and ethane more aromatic than benzene itself. Of course, the paradox is quickly dispelled by noting that the loop about the hydrogen atoms in $\mathrm{H}_{2} \mathrm{O}, \mathrm{NH}_{3}$, or $\mathrm{CH}_{4}$ has a much smaller radius than the radius of the benzene ring.

An ab initio method for treating open shell systems combined with an ab initio treatment of the paramagnetic chemical shifts has been proposed by Autschbach et al. (Gendron et al., 2015). Where spin-orbit coupling is not significant, it had been sufficient to use electron paramagnetic resonance (EPR) pseudospin Hamiltonian parameters ( $\mathrm{g}$ and $\mathrm{A}$ tensors, usually calculated using DFT methods) to calculate the contact and pseudocontact contributions to shielding in a paramagnetic molecule. The Fermi contact contribution arises from the isotropic part and the pseudo-contact contribution arises from the anisotropic part of the hyperfine coupling tensor. In the general case where spin-orbit coupling is strong, an ab initio theoretical approach that treats the open shell system that may have orbital degeneracies, low-lying excited states, in addition to strong spin-orbit coupling, is needed. Autschbach et al. (Martin and Autschbach, 2015) start from the concept proposed by Soncini et al. (Van den Heuvel and Soncini, 2013), that the temperature dependence of the shielding tensor could be defined by using the bi-linear derivative of the Helmholtz free energy with respect to the magnetic field and the nuclear magnetic moment. The operators which are the linear and bilinear derivatives of the Hamiltonian (with respect to the field $\mathbf{B}$, with respect to the nuclear moment $\boldsymbol{\mu}^{\mathrm{N}}$ and the bilinear derivative with respect to $\mathbf{B}$ and $\boldsymbol{\mu}^{\mathrm{N}}$ are expressed as usual and the temperature dependent shielding tensor from the Helmholtz free energy) is written in terms of these derivative operators. With the ensemble average carried out before the integration from 0 to $\beta\left(=1 / k_{B} T\right)$, subsequent integration leads to sum-over-states like expression for the shielding tensor components in terms of $Q$, the canonical partition function in the absence of the external field or the fields from nuclear moments. This expression is general in the sense that the wavefunctions and operators may include relativistic effects to all orders. Autschbach et al. find that contrary to conventional ideas, the temperature dependence of contact contribution to shielding is $1 / T$ and the pseudo-contact contribution to shielding is $1 / \mathrm{T}^{2}$ only in the cases where $\mathrm{S} \geq 1$ and zero-field splitting is appreciable, and only if $\mathrm{g}$ is isotropic (Martin and Autschbach, 2015). Otherwise the temperature dependence is not so simply separable for the two contributions. In a subsequent paper, Autschbach et al, (Gendron et al., 2015) start with the general expression in the sum-over-states form in the previous paper and consider the various parts. The operator, that is the derivative with respect to the nuclear moment of the nucleus of interest, has three contributions: Fermi contact, spindipole, and paramagnetic spin-orbital. The Fermi contact mechanism is isotropic and the contact shift arises from a net magnetization around and at the probe nucleus. One should note their use of the more general term net 
magnetization rather than "spin density", because in the general case both spin and orbital angular momenta are important, that is, contributions from all three mechanisms arise. When SO coupling is significant, then the association of the FC operator with contact paramagnetic shifts and spin-dipole operator with dipolar paramagnetic shifts no longer applies, and also, the electronic states are not pure spin multiplets. They explicitly state the conditions under which the general expression morphs into the commonly used equations in terms of EPR parameters: orbitally nondegenerate ground state, absence of lowenergy excited states, and relatively weak SO coupling. They consider an example where none of these explicit conditions apply: the paramagnetic shifts of ligand nuclei $\left({ }^{13} \mathrm{C}\right)$ in actinide complexes $\left[\mathrm{UO}_{2}\left(\mathrm{CO}_{3}\right)_{3}\right]^{3-}$ and $\left[\mathrm{NpO}_{2}\left(\mathrm{CO}_{3}\right)_{3}\right]^{4-}$. They use restrictive active space (RAS) calculations and include SO coupling in the actinide $5 \mathrm{f}$ shell via state interaction. They find that inclusion of higherlying unoccupied orbitals in the active space improves the convergence, especially for the Np complex (Gendron et al., 2015).

For systems where the spin-orbit coupling is weak, and excitations only within the ground state zero-field splitting manifold makes significant contribution, and the higher order zero field splitting and the higher order Zeeman terms can be neglected, then a nonrelativistic calculation such as proposed by Pennanen and Vaara (Pennanen and Vaara, 2008) could be used, provided that corrections to their original formulation are made, a flaw originally pointed out by Soncini et al., leading to an incorrect temperature dependence limiting behavior at low temperatures (Soncini and Van den Heuvel, 2013). Vaara et al. recently provide the corrected formulation, which now includes the magnetic couplings arising from the Zeeman and hyperfine coupling interactions between the states belonging to different degenerate manifolds (Rouf et al., 2015). They then apply the corrected theory to calculations of ${ }^{1} \mathrm{H}$ shifts in three paramagnetic systems of high spin Co(II) pyrazolylborate complexes. They find that using DFT calculations for geometry optimization and calculations of all tensors: $\mathbf{g}$, zero field splitting (D), and hyperfine $\mathbf{A}$, for the paramagnetic contributions, in addition to the usual (orbital) nuclear magnetic shielding with various functionals gave poor results, making it impossible to assign the protons correctly. Using $a b$ initio CASSCF for calculating the tensors $\mathbf{g}$ and $\mathbf{D}$, even with locally dense basis sets gave accurate results. The choice of basis functions is very important for accurate calculation of the $\mathbf{A}$ tensor and choice of functional does matter. A $25 \%$ admixture of exact exchange in these systems gives better results than other recipes.

As increased MAS spinning speeds became technically practical, it became possible to develop solid state NMR analogs of many solution state paramagnetic NMR experiments. The pseudocontact shift and paramagnetic relaxation enhancement are taken as dependent on the distances to and the orientations of the molecular paramagnetic susceptibility tensor arising from neighboring unpaired electrons. In the solid state, additional information is afforded by the anisotropy of the paramagnetic shift of each NMR active nucleus. These anisotropic paramagnetic shifts can be calculated via the general formalisms described above (Gendron et al., 2015), and the anisotropic tensor components provide more stringent tests of theory than isotropic shifts obtained from gases or solutions. At this point it is important to remember the caveats raised by Autschbach et al., that only under specific conditions is the pseudocontact shift dominantly from the dipolar mechanism and only under those conditions (spin-orbit coupling is weak, orbitally non-degenerate ground state, absence of low-energy excited states) may it be related to structure as commonly used. From the observed anisotropic shift tensors structural information is deduced, or starting structures are refined. Furthermore, the correlation of the paramagnetic shift tensor with other tensor quantities such as the quadrupolar coupling tensor can provide additional constraints. A recently developed method for correlating quadrupolar and paramagnetic shift interactions has been proposed by 
Grandinetti et al. that also enhances sensitivity and eliminates artifacts (Walder et al., 2015). They demonstrate the utility of these pulse sequences in obtaining accurate parameters for the ${ }^{2} \mathrm{H}$ paramagnetic shift and quadrupolar coupling tensors for the model compound $\mathrm{CuCl}_{2} \cdot 2 \mathrm{D}_{2} \mathrm{O}$. In a typical Grandinnetti approach, they then closely examine point dipole models for predicting the full paramagnetic shift tensor and propose a modified approach, guided by the shape of the singly occupied molecular orbital (SOMO), that yields excellent agreement with their measured results. While only a general theory, such as given above (Gendron et al., 2015), can include all possible relative magnitudes of contributions, thereby providing a systematic approach to accurate calculations for reproducing experimental quantities, a simple model is often useful to help us focus on the fundamental understanding that is possible in simple model systems. In this model system, their analysis of the full deuterium paramagnetic shift tensor reveals that a nucleicentered point dipole model, which delocalizes the unpaired spin density from the copper onto the two closest chlorides, yields poor agreement with their measured values, incorrectly predicting not only the sign of the paramagnetic shift anisotropy but also the tensor orientation. Agreement with the nucleicentered point dipole model is improved when the unpaired spin density is additionally delocalized onto the two oxygen ligands, yielding the correct sign of the paramagnetic shift anisotropy and closer agreement with the measured tensor orientation. Nearly perfect agreement within the uncertainty of their measurement, however, is found with a model with point dipoles displaced away from the nuclei and at positions consistent with the maximum electron density of the lobes of the singly occupied anti-bonding molecular orbital (Walder et al., 2015). Not only does this model yield excellent agreement, but it results in fractional delocalization of $13.3 \%$ onto the two closest chlorides and $1.6 \%$ onto the two closest oxygen ligands, a finding that is more consistent with results obtained from electron paramagnetic resonance and magnetic neutron scattering experiments on this system.
$\boldsymbol{A b}$ initio and DFT calculations. The $a b$ initio coupled-cluster $\operatorname{CCSD}(T)$ is the gold standard method for accurate quantum mechanical electronic structure and properties calculations, when full configuration interaction is not feasible. On the other hand, density functional method (DFT) is in widespread use for relativistic and nonrelativistic calculations, especially for large systems, despite the fact that there is no universal exchange -correlation functional that works uniformly well for all systems, even for a particular property such as nuclear magnetic shielding. A large number of functionals are in current use for this purpose, but for nonrelativistic calculations, most functionals do not perform well relative to the benchmark calculations using $\operatorname{CCSD}(T)$ for the identical molecular system. A comprehensive assessment of nuclear shielding calculations using DFT has been carried out by Gauss and co-workers against $\operatorname{CCSD}(\mathrm{T})$ calculations for relatively small molecular systems where gas phase absolute shielding data are also available for comparison (Teale et al., 2013). Fourcomponent relativistic calculations are not yet fully developed to include electron correlation at the CCSD(T) level. Thus, DFT (with some electron correlation) and Hartree-Fock (without electron correlation, but with exact exchange) calculations are used.

Accurate non-relativistic calculations of absolute shielding and spin-rotation tensors for fluorine-containing diatomic molecules XF (where $\mathrm{X}=\mathrm{B}, \mathrm{Al}, \mathrm{Ga}$, In, $\mathrm{Tl}$ ) using $\operatorname{CCSD}(\mathrm{T})$ for $\mathrm{BF}, \mathrm{AlF}$, and $\mathrm{GaF}$ and CCSD for InF and $\mathrm{TlF}$, and relativistic corrections from fourcomponent relativistic density-functional theory (using the PBE functional ) added together with rovibrational averaging, are seen to provide good agreement with experimental values, where available (Jaszunski et al., 2014). The restricted magnetic balance scheme was used for the nuclear magnetic shielding tensor calculations, whereas the restricted kinetic balance scheme was used for spin-rotation. The common gauge-origin approach was used in the four-component calculations of both spin-rotation and absolute shielding. As expected, the relativistic effects play an 
increasingly important role as one proceeds to the heavier elements in the Periodic Table. The calculated values for the spans, $\Omega\left({ }^{115} \mathrm{In}\right)$ and $\Omega\left({ }^{205} \mathrm{Tl}\right)$ are 1805 and $7136 \mathrm{ppm}$, respectively, showing the importance of the relativistic effects in determining the shielding anisotropy. For example, the previously reported $\Omega\left({ }^{205} \mathrm{Tl}\right)$ based on the experimental spin-rotation constant and the Ramsey nonrelativistic identity relation (Ramsey, 1950) ${ }^{11}$ is smaller by $26 \%$ than the relativistically corrected value. This example affirms the need for the relativistic mapping of spinrotation and nuclear magnetic shielding, as derived by Liu et al. (Xiao et al., 2012). Overall, for the light nuclei there is good agreement between the calculated values and experimental data, which is not the case for the heavier nuclei; $\Omega\left({ }^{115} \mathrm{In}\right)$ is off by $145 \mathrm{ppm}$ and $\Omega\left({ }^{205} \mathrm{Tl}\right)$ by $1840 \mathrm{ppm}$. Even for $\Omega\left({ }^{19} \mathrm{~F}\right)$ the difference between experimental and calculated values increases from $4 \mathrm{ppm}$ to 43 ppm with increasing atomic number of the other atom.

Relativistic 4-component calculations using polarization propagators at the RPA level (uncorrelated) are compared against 4component DFT (using 6 different functionals) for ${ }^{207} \mathrm{~Pb}$ and ${ }^{119} \mathrm{Sn}$ in the $\mathrm{Sn}$ and $\mathrm{Pb}$ halides ( $\mathrm{Sn}$ or $\mathrm{Pb}) \mathrm{Y}_{4 \mathrm{n}} \mathrm{Z}_{\mathrm{n}}(\mathrm{Y}, \mathrm{Z}=\mathrm{H}, \mathrm{F}, \mathrm{Cl}, \mathrm{Br}, \mathrm{I})$ (Maldonado and Aucar, 2014). Using 2component LRESC (a relativistic linear response theory with elimination the two small components) approach, calculations of Sn, Ge, and $\mathrm{Si}$ shielding in molecules with halogen ligands (halo $=\mathrm{F}, \mathrm{Cl}, \mathrm{Br}, \mathrm{I}$ ) are compared against 4-component relativistic polarization propagator formalism at the RPA level. Both the calculation of non-relativistic and the leading LRESC contributions to the shielding were carried out at RPA level, the gauge origin placed at the nucleus of interest for all LRESC calculations (Maldonado et al., 2014).

A careful analysis of neighbor effects in relativistic systems is reported for ${ }^{199} \mathrm{Hg}$ shielding tensors in 11 solids, from $\mathrm{HgF}_{2}$ to $\mathrm{Hg}_{2}\left(\mathrm{NO}_{3}\right)_{2} \cdot 2 \mathrm{H}_{2} \mathrm{O}$, using various molecular cluster systems for the structures used in shielding calculations (Alkan and Dybowski,
2014). Careful design of a cluster to represent the solid-state structure, inclusion of relativistic components in the Hamiltonian at least at the spin-orbit level (in this case using DFT-ZORA, a popular method using zerothorder regular approximation), and judicious use of approximations are essential to obtain good agreement with experimental results. It is of course essential to have the correct site symmetry at the nucleus, especially when calculating individual tensor components rather than isotropic averages. The largest neutral cluster that is feasible and has the correct site symmetry at the nucleus of interest is arrived at starting from the first coordination shell and growing outward to include the next coordination shell. The frozen core approximation may be used for the remotest atoms, with their inner core of electrons treated relativistically. Total neglect of relativistic terms is the poorest approximation; inclusion of scalar relativistic terms improves the calculation slightly, but for the highest accuracy, one must include spin-orbit components in the Hamiltonian. This molecular cluster approach was further applied to solid state NMR of ${ }^{207} \mathrm{~Pb}$ and ${ }^{119} \mathrm{Sn}$ in the pigment leadtin yellow type I that had been used until the first half of the $18^{\text {th }}$ century (Catalano et al., 2014). The calculations were used to identify the individual chemical species in the pigment.

Spin orbit contributions to ${ }^{13} \mathrm{C}$ and ${ }^{15} \mathrm{~N}$ shielding in $\mathrm{Pt}$ and $\mathrm{Au}$ complexes were calculated using sum-over-states finite-field third-order perturbation theory with scalar relativistic effective core potentials for Pt and $\mathrm{Au}$ and are compared with ZORA-SO calculations (Vicha et al., 2015). Analysis in terms of the atoms-in-molecules approach is offered for understanding the effect of heavy atoms on light atom shielding. Calculations of ${ }^{13} \mathrm{C}$ shielding in dihaloethenes (halo $=\mathrm{F}, \mathrm{Cl}$, $\mathrm{Br}, \mathrm{I})$ using DFT-ZORA including SO (and using the KT2 functional) are analyzed in $\mathrm{NBO} / \mathrm{MO}$ terms to understand the differences between cis and trans isomers (Viesser et al., 2015). Using natural bond orbitals (NBO) analysis permits an intuitive view using the familiar MOs that chemists are most 
comfortable with. In a separate study of dihaloethenes, the principal factor responsible for the difference (17 ppm) in the ${ }^{13} \mathrm{C}$ NMR chemical shift between the cis- and transdiiodoethene isomers was the steric interaction observed between the two iodine atoms in the cis isomer. DFT-ZORA calculations with $\mathrm{SO}$ of ${ }^{19} \mathrm{~F}$ shielding in 20 fluorinated organic molecules used the BLYP functional. Even with the $\mathrm{Cl}-$ and $\mathrm{Br}-$ substituted organic groups, the relativistic contributions are found to be modest (Saielli et al., Computational, 2014).

MP2 and DFT calculations have been reported for ${ }^{31} \mathrm{P}$ shielding in 51 different organophosphorus compounds (Fedorov et al., 2014) using various functionals, with the best results arrived at using the functionals of Keal and Tozer, KT2 and KT3 (Keal and Tozer, 2003; Keal and Tozer, 2004). MP2 (up to second order in the Møller-Plesset perturbation theory) occupies a unique role in quantum chemistry due to its unusually cheap $a b$ initio treatment of electron correlation. The locally dense basis set approach results in a further dramatic decrease in computational cost without loss of accuracy. Solvation effects are modeled by polarizable continuum model (PCM). Relativistic corrections, calculated using 4-component DFT are 20-30 ppm.

DFT non-relativistic, relativistic scalar ZORA, and spin-orbit ZORA calculations of ${ }^{15} \mathrm{~N}$ shielding in complexes of rhodium(II) tetraformate with 11 organic ligands used PCM for solvent effects (Leniak and Jazwinski, 2015). Correlation coefficients for shielding versus experimental chemical shifts had slopes equal to $\sim 0.87$ (ideally should be 1.0) for various functionals used when all 11 ligands are included in the plot. The ${ }^{77} \mathrm{Se}$ shielding in 28 selenium-containing molecules, for which the ${ }^{77} \mathrm{Se}$ chemical shifts are unambiguously known, were calculated to help in assignment of ${ }^{77} \mathrm{Se}$ in cyclic selenium imides 1,3,5,7-Se ${ }_{4}(\mathrm{NMe})_{4}$ puckered crownshaped molecules and in other cyclic imides $\mathrm{Se}_{6}(\mathrm{NMe})_{2}$ and disordered cyclo $\mathrm{Se}_{8}$ molecules (Karhu et al., 2015). First of all, solvent effects were not modeled in any way (DFT calculations of single molecule in vacuum); furthermore, some relativistic corrections for this moderately heavy atom should have been included.

Non-relativistic DFT calculations of shielding for light atoms, usually with PCM standing in for the solvent, provide quick resolution of spectral assignments and answers to structural questions for experimentalists. Some recent examples are the following: DFT calculation of ${ }^{15} \mathrm{~N}$ NMR chemical shifts of the nitrogencontaining heterocycles (best results with KT3 functional) (Samultsev et al., 2014), using the KT3 functional and PCM for ${ }^{15} \mathrm{~N}$ NMR chemical shifts of 27 azoles and azines in 10 different solvents (Semenov et al., 2014), using mPW1PW91 functional for calculations of ${ }^{1} \mathrm{H}$ chemical shifts in lactones (Aimola et al., 2015), using B3LYP for ${ }^{1} \mathrm{H}$ and ${ }^{13} \mathrm{C}$ in tautomeric 1-[(pyridin-2-yl amino) methyl] pyrrolidine-2,5-dione (Boobalan et al., 2014), using mPW1PW91 and PCM for ${ }^{1} \mathrm{H}$ and ${ }^{13} \mathrm{C}$ in lignin amino acid adducts (Diehl et al., 2014), using 16 different functionals for ${ }^{1} \mathrm{H}$ and ${ }^{13} \mathrm{C}$ in (R)-ispinesib, a drug molecule (Hill et al., 2015), using B3LYP in COSMO solvent model for for ${ }^{1} \mathrm{H}$ and ${ }^{13} \mathrm{C}$ shielding in bis $(4$ trimethylammoniumbenzoate) hydroiodide hydrate in DMSO (Komasa et al., 2015), using B3LYP for ${ }^{13} \mathrm{C}$ shielding in tautomers of vitamin B-13 in PCM solvent model (Kubica and Gryff-Keller, 2015), using 7 different functionals for ${ }^{13} \mathrm{C}$ shielding in 2hydroxylamino-4,6-dinitrotoluene (Liu et al., 2015), using BP86 functional for ${ }^{13} \mathrm{C}$ shielding tensors in $\mathrm{C}_{60}$ and $\mathrm{C}_{60}{ }^{10+}$ (Munoz-Castro, 2015), using $\mathrm{BHandH}$ functional for ${ }^{13} \mathrm{C}$ and ${ }^{17} \mathrm{O}$ in 1,3-dioxolane and ${ }^{19} \mathrm{~F}$ in perfluoro-1,3dioxolane (Nozirov et al., 2014), using B3LYP for ${ }^{1} \mathrm{H}$ and ${ }^{13} \mathrm{C}$ in in 2-bromo-1H-benzimidazol (Sas et al., 2015), using B3LYP for ${ }^{1} \mathrm{H}$ and ${ }^{13} \mathrm{C}$ shielding in $1: 2$ complex of dimethylphenyl betaine with 2,6-dichloro-4nitro-phenol in DMSO (Szafran et al., 2015), and using the MPW1K (Lynch et al., 2000) hybrid functional with PCM solvent model for ${ }^{29} \mathrm{Si}$ in 24 organosilanes (Zhang et al., 2014). A review article on addressing the stereochemistry of complex organic molecules by density functional theory calculations of NMR 
parameters, nuclear shielding in particular, has appeared (Bagno and Saielli, 2015).

DFT/B3LYP calculations of ${ }^{13} \mathrm{C}$ and ${ }^{17} \mathrm{O}$ shielding in vitamin $\mathrm{E}$ models in chloroform and in water suggest that a proper description of the solvent shift for ${ }^{17} \mathrm{O}$ nuclear magnetic shielding of the hydroxyl group in water requires the use of explicit solute-solvent hydrogen bonds (Oliveira et al., 2015). The association of two small molecules is studied by DFT calculations using IGLO-II basis set for the ${ }^{11} \mathrm{~B}$ and ${ }^{15} \mathrm{~N}$ shielding in $\mathrm{NH}_{3} \mathrm{BH}_{3}$ (Kobayashi et al., 2014). This provided insights into the dehydrogenation mechanism of $\mathrm{NH}_{3} \mathrm{BH}_{3}$, insights that have not been available through ${ }^{11} \mathrm{~B}$ NMR alone.

In a study of ${ }^{17} \mathrm{O}$ in solid $\mathrm{N}$-acyl imidazoles, quantum chemical computations show that the dependence of ${ }^{17} \mathrm{O}$ NMR parameters on the $\operatorname{Ar}-\mathrm{C}(\mathrm{O})$ bond rotation is very similar to that previously observed for the $\mathrm{C}(\mathrm{O})-\mathrm{N}$ bond rotation in twisted amides. The authors conclude that one should be cautious in linking the observed NMR chemical shifts only to the twist of the $\mathrm{C}(\mathrm{O})-\mathrm{N}$ amide bond (Kong et al., 2015).

In a study of disiline-doped $(6,0)$ zigzag singlewalled boron nitride nanotubes, the shielding values of ${ }^{11} \mathrm{~B}$ and ${ }^{15} \mathrm{~N}$ nuclei were calculated using three different ways of taking care of the gauge problem that arises when using incomplete basis sets: by Gauge-Including Atomic Orbital (GIAO), Continuous Set of Gauge Transformations (CSGT) and Individual Gauges for Atoms in Molecules (IGAIM) methods. The authors used the B3LYP functional and a hydrogen-terminated section of nanotube model $\left(\mathrm{B}_{14} \mathrm{~N}_{14} \mathrm{H}_{8}\right)$ (Arshadi et al., 2014). The disiline doping is modeled by $\mathrm{C}_{4} \mathrm{Si}_{2}$ replacing the three $\mathrm{B}$ and three $\mathrm{N}$ atoms in a ring in the model. The results reveal a significant effect of disiline doping on the nuclear shielding tensors at the sites of those ${ }^{11} \mathrm{~B}$ and ${ }^{15} \mathrm{~N}$ nuclei located in the nearest neighborhood of the disiline-doped ring. In another study, DFT-B3LYP calculations on covalent addition of homoand hetero-diatomic molecules $(\mathrm{AB})$ including $\mathrm{H}_{2}, \mathrm{O}_{2}, \mathrm{~N}_{2}, \mathrm{NO}$ and $\mathrm{CO}$ at the external surface of $\mathrm{H}$-capped pristine armchair $(5,5)$ single-walled carbon nanotube (SWCNT) were conducted (Jankowska et al., 2015). The SWNT is modeled by an $\mathrm{H}$-terminated section $\left(\mathrm{C}_{70} \mathrm{H}_{10}\right)$ and the corresponding $\mathrm{C}_{70} \mathrm{H}_{10}-\mathrm{AB}$ covalent adducts were fully optimized at the B3LYP/6-311G* level of theory. Significant changes of carbon NMR atom chemical shifts (up to $-100 \mathrm{ppm}$ ) and shielding anisotropies (up to $-180 \mathrm{ppm}$ ) at sites of addition (chosen as C3 and C4 positions from the hydrogen terminated end) were calculated. This is not at all the "adsorption" of gases that it is erroneously called in the abstract.

\section{PHYSICAL ASPECTS OF NUCLEAR SHIELDING}

Anisotropy of the Shielding Tensor. The study of nuclear shielding includes advances in our understanding of what and how various factors affect this electronic property as well as practical improvements in measurements and theoretical predictions. The various papers recently published in this area are now either tackling deeper details by introducing better experimental and theoretical techniques, or finding new applications by unraveling new correlations for this ubiquitous NMR parameter.

Proton shielding tensors are inherently difficult to determine accurately. The inhomogeneity of the radiofrequency field sometimes impairs recoupling sequences in solid state NMR measurements. This difficulty often manifests in experiments geared toward the determination of proton chemical shift tensors. Through the incorporation of a series of phase-alternating $270_{0}-90_{180}$-composite- 180 pulses, inhomogeneity effects can be significantly reduced, as demonstrated in the measurements of proton shielding tensors in citric acid and malonic acid (Pandey et al., 2015). The proton shielding tensor for water in barium chlorate monohydrate has been determined via solid state NMR under both static and magic-angle spinning conditions. A two-dimensional technique has likewise been employed to separate the shielding tensor from the dipolar interaction. Both techniques yield similar anisotropy and asymmetry. 
Calculated values from plane wave calculations are made to agree with experiment only upon incorporation of motional averaging caused by the fast rotation of the water molecule about its $\mathrm{C}_{2}$ axis (Carnevale et al., 2014). With a magnetic field of $19.9 \mathrm{~T}$ and MAS frequency of $62 \mathrm{kHz}$, three dimensional heteronucleus-detected dipolar based solid state NMR experiments have been used to observe and assign backbone resonances for the 89-residue dynein light chain 8, LC8 protein (Guo et al., 2014). In this scheme, the fast spinning allows for ${ }^{1} \mathrm{H}$ resonances to be included, which aids greatly in making assignments, while direct observation of ${ }^{13} \mathrm{C}$ provides greater resolution.

The type of secondary helix structure $\operatorname{poly}(\beta$ benzyl L-aspartate) assumes in the solid state depends on its molecular weight and removal rate of the solvent thereby paving the way to examine both right-handed and left-handed helices. Employing the Switching-Angle Sample-Spinning (SASS) method, the carbonyl ${ }^{13} \mathrm{C}$ shielding tensors have been recently measured. It is found that the intermediate component of the carbonyl shielding tensor is very sensitive to the handedness of the helix. This is only expected as the $\mathrm{C}=\mathrm{O}$ group begins to interact with the sidechain when a peptide assumes a left-handed helical conformation. The experimental $\delta_{22}$ values in the right-handed $\alpha_{R}$, and left-handed $\alpha_{L}$ and $\omega_{\mathrm{L}}$ samples are 184, 174, and $164 \mathrm{ppm}$, respectively (Kamihara et al., 2015). Restraints derived from ${ }^{15} \mathrm{~N}$ chemical shift anisotropy have been shown to improve the structure of the bacterial mercury transporter protein MerF (Tian et al., 2014). Lastly, measurements of cross-correlation effects between dipolar $\left({ }^{1} \mathrm{H}^{\mathrm{N}}-{ }^{1} \mathrm{H}^{\alpha}\right)$ and amide-proton chemical shift anisotropy ( ${ }^{1} \mathrm{HN}$ CSA) on longitudinal relaxation times of the amide proton in a model tripeptide $\mathrm{Piv}^{-}-{ }^{\mathrm{L}} \mathrm{Pro}-{ }^{\mathrm{L}} \mathrm{Pro}-{ }^{\mathrm{L}} \mathrm{Phe}-\mathrm{OMe}$ have shown that hydrogen bonding increases the anisotropy of the amide proton shielding tensor (Pichumani et al., 2015).

To obtain both magnitude and orientation of the shielding tensor components, a single crystal is required. Kusumi and coworkers have demonstrated that this can be equally achieved in the case of the ${ }^{31} \mathrm{P}$ chemical shift tensor in phenyl-phosphonic acid (PPA) by making use of a sample of PPA microcrystals aligned three-dimensionally in a polymer matrix (Kusumi et al., 2015). With new ${ }^{43} \mathrm{Ca}$ double-rotation (DOR) and multiple-quantum magic-angle spinning (MQMAS) spectra and powder diffraction data, combined with computer-generated structural models and chemical shift tensor and electric field gradient calculations, possible structures for the vaterite polymorph of $\mathrm{CaCO}_{3}$ have been narrowed down to two candidates, hexagonal $\mathrm{P}_{2} 21$ and monoclinic $\mathrm{C} 2$ (Burgess and Bryce, 2015). NMR parameters for ${ }^{13} \mathrm{C},{ }^{15} \mathrm{~N}$, and ${ }^{51} \mathrm{~V}$ have been successfully tapped in combination with model calculations to determine in greater detail the structure in the solid state of [VO $\left.\left({ }^{15} \mathrm{NGlySal}\right)\left(\mathrm{OCH}_{3}\right)\left(\mathrm{CH}_{3} \mathrm{OH}\right)\right]$, a compound that is now known to have a methanol molecule coordinated to vanadium ( $\mathrm{Li}$ et al., 2015). ${ }^{35} \mathrm{Cl}$ electric field gradient (EFG) and chemical shift tensors have been measured in a series of chlorine-containing transition metal complexes (O'Keefe et al., 2014). It is observed that the observed EFG tensor is quite sensitive to whether the chlorine ligand is bridging, terminal-axial or terminalequatorial. An experimental and theoretical NMR study of ${ }^{185 / 187} \mathrm{Re}$ has been carried out on several perrhenates $\left(\mathrm{KReO}_{4}, \mathrm{AgReO}_{4}\right.$, $\left.\mathrm{Ca}\left(\mathrm{ReO}_{4}\right)_{22} \mathrm{H}_{2} \mathrm{O}\right), \quad \mathrm{ReO}_{3}, \quad$ and $\operatorname{Re}_{2}(\mathrm{CO})_{10}$ (Widdifield et al., 2015). The ${ }^{77} \mathrm{Se}$ chemical shift tensor for L-selenocystine has also been measured in the solid state (Struppe et al., 2015). The observed isotropic value in the solid state is about $50 \mathrm{ppm}$ more shielded compared to what is seen in solution. Model calculations reveal that this difference is likely a consequence of the dramatic sensitivity of the ${ }^{77} \mathrm{Se}$ shielding on the $\mathrm{C}-\mathrm{Se}-\mathrm{Se}-\mathrm{C}$ dihedral angle. This dihedral angle can change the ${ }^{77} \mathrm{Se}$ shielding by as much as $600 \mathrm{ppm}$.

\section{Shielding Surfaces and Rovibrational} Averaging. Even in the solid state, atoms are still in thermal motion. An accurate comparison between calculated and experimental chemical shifts must therefore go beyond a static lattice approximation. Due 
to the large number of vibrational modes, incorporation of dynamics in shielding calculations in the solid phase imposes high demands on computational resources and time. For instance, Monte Carlo simulations often require hundreds of sampling points at any given temperature. On the other hand, shielding surfaces constructed by a finite displacement method require much less sampling points. With this scheme, Monserrat and coworkers have been able to compute vibrational corrections to shieldings in magnesium oxide and the molecular crystals Lalanine and $\beta$-aspartyl-L-alanine (Monserrat et al., 2014). Results obtained from the constructed shielding surfaces are as accurate as those obtained via Monte Carlo simulations. Since quadratic and anharmonic contributions are obtained separately when the shielding surfaces are used, it is likewise straightforward to see that thermal corrections to shielding in solids are dominated by the quadratic terms.

The conformational flexibility of saccharides is an absolute requirement for the molecular recognition by biological targets, such as enzymes and antibodies. Therefore, structural determination is crucial for understanding these recognition processes. The application of DFT calculations for the NMR determination of the conformation of $\beta$-cellobiose in water has been carried out (Yan and Yao, 2015). The cellobiose flexibility mainly arises from two dihedral angles, $\phi\left(\mathrm{H}^{\prime}-\mathrm{C1}^{\prime}-\mathrm{O}\right.$ '-C4) and $\psi\left(\mathrm{C}^{\prime}-\mathrm{O}\right.$ '-C4-H4), which in principle vary from $-180^{\circ}$ to $+180^{\circ}$. The authors employed several different functionals and used PCM for solvent model. The PW1PW91 functional is found to be the best with a correlation slope of 1.026 and 1.008 for ${ }^{13} \mathrm{C}$ and ${ }^{1} \mathrm{H}$ chemical shifts, respectively. They report ${ }^{13} \mathrm{C}$ NMR chemical shifts for $\mathrm{C} 4$ and $\mathrm{C}$ ' at three energy-minimum conformations: syn$\phi / \operatorname{syn}-\psi\left(\phi=32.2^{\circ}, \psi=\sim 23.9^{\circ}\right)$, anti- $\phi /$ syn $-\psi$ $\left(\phi=178.2^{\circ}, \psi=\sim 0.4^{\circ}\right)$, and syn- $\phi /$ anti- $\psi$ $\left(\phi=10.5^{\circ}, \psi \sim 180^{\circ}\right)$. The calculations suggest that the $\mathrm{C1}$ ' chemical shifts are sensitive to the conformational changes, with the values of $103.3 \mathrm{ppm}$ at $s y n-\phi / s y n-\psi, 98.9 \mathrm{ppm}$ at anti- $\phi / s y n-\psi$, and $95.3 \mathrm{ppm}$ at syn- $\phi /$ anti- $\psi$. Likewise, the C4 chemical shifts are sensitive to the conformational changes, with the values of $80.4 \mathrm{ppm}$ at $s y n-\phi / s y n-\psi, 70.0 \mathrm{ppm}$ at anti- $\phi / s y n-\psi$, and $75.6 \mathrm{ppm}$ at syn- $\phi /$ anti- $\psi$. The ${ }^{13} \mathrm{C}$ chemical shifts' ability to distinguish between characteristic positions is helpful for the determination of conformations, especially for those with equivalent energies. The shielding tensor analysis shows that for C1', the directions and amplitudes of two shielding components $\left(\sigma_{22}\right.$ and $\left.\sigma_{33}\right)$ vary distinctly with $\phi$ and $\psi$, whereas for $\mathrm{C} 4, \sigma_{33}$ remains unchanged, but $\sigma_{22}$ varies by 13 ppm from syn$\phi / s y n-\psi$ to anti- $\phi / s y n-\psi$ (syn- $\phi /$ anti- $\psi$ ). (For the $\mathrm{C} 1$ ' the corresponding value is $26 \mathrm{ppm}$ ). The distinctive C4 and C1' chemical shifts at different conformations suggest that they can be useful in $(\phi, \psi)$ dihedral angle determinations (Yan and Yao, 2015). The dependence of ${ }^{13} \mathrm{C}$ shieldings on the glycosidic torsional angles $(\phi, \psi)$ has also been calculated using a disaccharide model (Garay et al., 2014). As expected, shieldings at the carbon sites participating in the glycosidic linkage are most sensitive to this conformational angle, exhibiting a range of about $20 \mathrm{ppm}$. This ${ }^{13} \mathrm{C}$ shielding surface is found to depend on several factors such as the identity of the disaccharide, the anomeric state, and the conformation of a nearby hydroxyl group. It is then estimated that before ${ }^{13} \mathrm{C}$ shieldings can be accurately used for validation, determination, and refinement of glycan structures, more than the 20,000 shielding computations are necessary.

Model clusters containing up to 20 [P(OAl$\left.\left.(\mathrm{OH})_{3}\right)_{4}\right]^{3-}$ units have been employed to extract computationally the dependence of ${ }^{31} \mathrm{P}$ shielding on $\mathrm{P}-\mathrm{O}-\mathrm{Al}$ bond angles and $\mathrm{P}-\mathrm{O}$ bond lengths. With these constructed shielding surfaces, successful predictions of ${ }^{31} \mathrm{P}$ chemical shifts in calcined aluminophosphates have been attained (Dawson and Ashbrook, 2014). The ${ }^{23} \mathrm{Na}$ shielding is presumed to be a function of $\mathrm{Na}-\mathrm{O}$ distances in silicate glasses. A linear relationship between ${ }^{23} \mathrm{Na}$ chemical shifts and $\mathrm{Na}-\mathrm{O}$ distances, however, becomes evident only 
when coordination number is carefully taken into account (Gambuzzi et al., 2014). A Boltzmann weighing of ${ }^{17} \mathrm{O}$ shielding from four low-energy structures of brownmilleritetype phase $\mathrm{Ba}_{2} \mathrm{In}_{2} \mathrm{O}_{5}, \mathrm{Ba}_{2} \mathrm{In}_{2} \mathrm{O}_{4}(\mathrm{OH})_{2}$, is shown to reproduce the observed solid state ${ }^{17} \mathrm{O}$ NMR spectra obtained from these perovskites (Dervisoglu et al., 2015). In this hydrated form of brownmillerite, the oxygen nuclei of hydrogen bond donors are shown to be shielded while both nonhydroxyl and hydrogen bond acceptor oxygen sites are found to be deshielded.

${ }^{29} \mathrm{Si}$ shielding traces with respect to the $\mathrm{Si}-\mathrm{O}-\mathrm{Si}$ bond angle have been constructed for three different tetrahedral environments $\{\mathrm{Si}\} \mathrm{O}_{4}$, $\{\mathrm{Si}\} \mathrm{O}_{3} \mathrm{C}$, and $\{\mathrm{Si}\} \mathrm{O}_{2} \mathrm{C}_{2}$ (Nimmo II and Kroll, 2014). With these traces, Si-O-Si angle distributions can be extracted from the ${ }^{29} \mathrm{Si}$ NMR spectra of amorphous silicon oxycarbide ceramics. Results derived from this approach are in agreement with those obtained from x-ray and neutron diffraction data. The dependence of ${ }^{29} \mathrm{Si}$ NMR chemical shifts on the ${ }^{\mathrm{IV}} \mathrm{B} /{ }^{\mathrm{III}} \mathrm{B}$ ratio in borosilicate glass has been utilized to characterize the structural effects of europium on these materials (Bouty et al., 2014). Similarly, ${ }^{29} \mathrm{Si}$ chemical shifts, being dependent on local structure and less sensitive to long-range order, have been demonstrated to be useful in the determination of the layered framework structure of the new silicate material CLS-1 of composition $\left[\mathrm{Si}_{5} \mathrm{O}_{11} \mathrm{H}\right]\left[\mathrm{C}_{9} \mathrm{~N}_{2} \mathrm{H}_{15}\right] \cdot 1.9\left(\mathrm{H}_{2} \mathrm{O}\right)$ (Cadars et al., 2014).

${ }^{31} \mathrm{P}$ solid state NMR spectra combined with two-dimensional experiments have been employed in characterizing a series of $\left(\mathrm{NaPO}_{3}\right)_{1-\mathrm{x}}\left(\mathrm{Ga}_{2} \mathrm{O}_{3}\right)_{\mathrm{x}}$ glasses $(0 \leq \mathrm{x} \leq 0.35)$ (Ren and Eckert, 2014). Here, ${ }^{31} \mathrm{P}$ spectra are deconvoluted to provide phosphorus speciations. ${ }^{31} \mathrm{P}$ chemical shifts are known to be sensitive to the identity of the next neighbor atom in phosphates as illustrated in the case of $\mathrm{Mg}^{2+}$ and $\mathrm{Zn}^{2+}$-substituted aluminophosphates (Seymour et al., 2015). In this particular case, the ${ }^{31} \mathrm{P}$ chemical shift is found to be significantly influenced by the P$\mathrm{O}-\mathrm{M}$ bond angle.
A combination of molecular dynamics simulation and plane wave shielding calculation has been applied to reproduce ${ }^{77} \mathrm{Se}$ NMR spectra of selenium-rich $\mathrm{Ge}_{x} \mathrm{Se}_{(1-x)}$ glasses (Sykina et al., 2014). Agreement with experimental spectra in this particular case is taken as a validation of the molecular dynamics simulation. ${ }^{207} \mathrm{~Pb}$ chemical shifts, especially their tensor span, are sensitive to the coordination number around $\mathrm{Pb}$. In a series of lead(II) carboxylates, it is found that with short chain carboxylates (octanoate or smaller), ${ }^{207} \mathrm{~Pb}$ tensor spans are more than $2500 \mathrm{ppm}$. With nonanoate or longer carboxylates, the span is reduced to $740 \mathrm{ppm}$, suggesting a closer to being spherical local structure at the lead atom site (Catalano et al., 2015).

${ }^{1} \mathrm{H}$ chemical shifts have been used with powder diffraction and theoretical calculations to arrive at possible structures for a substituted zeolitic imidazolate metal-organic framework (Baias et al., 2015). In these systems, ${ }^{1} \mathrm{H}$ chemical shifts are predicted to be sensitive to the relative orientation of aldehyde and aromatic groups. Computed ${ }^{1} \mathrm{H}$, ${ }^{13} \mathrm{C}$ and ${ }^{31} \mathrm{P}$ chemical shifts using optimized crystal structures are found to be helpful in a further refinement of the solid state structure of phospho-tyrosine (Paluch et al., 2015), and plane wave shielding calculations are found to be of value in assigning the ${ }^{13} \mathrm{C}$ and ${ }^{15} \mathrm{~N}$ resonances observed in polycrystalline materials containing co-crystals of theophylline with malonic, maleic and glutaric acids (Pindelska et al., 2014). The ${ }^{1} \mathrm{H}$ and ${ }^{13} \mathrm{C}$ chemical shifts have been successfully predicted for the four crystal forms $(1 \alpha, 1$. $\mathrm{H}_{2} \mathrm{O}, 1 \beta$, and $1 \gamma$ ) of the solid wheel-and-axle metal-organic compound [(p-cymene)$\mathrm{Ru}(\kappa \mathrm{N}-\mathrm{INA}) \mathrm{Cl}_{2}$ ] (Presti et al., 2014), and plane wave calculations of ${ }^{13} \mathrm{C}$ chemical shifts can likewise be employed to validate an $\mathrm{x}$-ray structure of a polymorph as demonstrated in the case of clopidogrel hydrogensulfate (Pindelska et al., 2015). The ${ }^{43} \mathrm{Ca},{ }^{13} \mathrm{C}$, and ${ }^{17} \mathrm{O}$ NMR chemical shifts have been calculated for bulk and surface atoms in calcite. In addition, the ${ }^{43} \mathrm{Ca}$ chemical shifts have been determined to be sensitive to hydrocarbon adsorption (Bevilaqua et al., 2014). Lastly, by including 
proton jumps and discriminating phosphate group rotations, ${ }^{17} \mathrm{O}$ NMR spectra at various temperatures can be used to characterize in greater detail the dynamics in the protonic conductor $\mathrm{CsH}_{2} \mathrm{PO}_{4}$ (Kim et al., 2015).

NMR crystallography is defined as the combined use of experimental NMR and computation to provide new insight, with atomic resolution, into structure, disorder and dynamics in the solid state. In this context, calculations of NMR parameters using the GIPAW (gauge-including projector augmented wave) method have been performed for a wide variety of organic solids. GIPAW calculations for the hemihydrate of 2', 3'-Oisopropylidineguanosine, whose crystal structure has been solved by single crystal x-ray diffraction, were performed with the CASTEP code using the PBE functional with a dispersion correction scheme (Reddy et al., 2015). A plane-wave basis set with ultra-soft pseudopotentials was used. Comparing the experimental and GIPAW calculated ${ }^{1} \mathrm{H}$ chemical shift, it is evident that there are greater discrepancies for the protons bonded to nitrogen than for protons bonded to carbon, notably the calculated $\mathrm{NH}_{2 \mathrm{~b}}{ }^{1} \mathrm{H}$ chemical shifts are significantly less than the experimental values. The authors suggest a possible explanation is the known temperature dependence of hydrogen-bonded protons, while the GIPAW calculation is performed for a static structure at a temperature of absolute zero. Another explanation is that $\mathrm{H}$ shielding in bonds to $\mathrm{C}$ is in general more permissive of the basis sets and electron correlation description than is $\mathrm{H}$ shielding in bonds to $\mathrm{O}$ or $\mathrm{N}$.

GIPAW calculations and a detailed analysis using complementary $1 \mathrm{D}$ and 2D SSNMR methods using ${ }^{1} \mathrm{H},{ }^{19} \mathrm{~F},{ }^{15} \mathrm{~N},{ }^{13} \mathrm{C}$ and ${ }^{35} \mathrm{Cl}$ nuclei have been conducted on a novel crystalline form of ronacaleret hydrochloride, a calciumsensing receptor antagonist under development for the treatment of osteoporosis. The paper serves as a paradigm for the comprehensive application of experimental and computational methods to a complex but typical drug in the solid phase (Vogt et al., 2014). CASTEP code with PBE was used for structure refinement and shielding calculations for all nuclei, in addition to quadrupole coupling tensor for ${ }^{35} \mathrm{Cl}$. The GIPAW calculation performed on the room temperature structure was found to lead to slightly more accurate reproduction of many NMR properties of interest in the present case than the low temperature structure obtained from x-ray diffraction.

A correspondingly comprehensive combined experimental-computational effort to characterize the $\mathrm{Al}$ surface sites of $\gamma$-alumina was undertaken (Wischert et al., 2014). The authors calculated ${ }^{27} \mathrm{Al}$ NMR parameters from first principles in periodic boundary conditions using the VASP code for structural optimization with a PW91 functional and CASTEP code using PBE functional for the NMR shielding and nuclear quadrupole coupling tensors. They did these calculations for a large number of $\mathrm{Al}$ sites with different coordination schemes, potentially present on the alumina surface. They calculated NMR parameters for all terminations, surface sites, and hydration levels. The nature and accordingly the NMR parameters of these sites change with the level of hydroxylation and thereby change with the pretreatment temperature of $\gamma-\mathrm{Al}_{2} \mathrm{O}_{3}$. Hydration leads to addition of $\mathrm{O}$ atom(s) to the first coordination sphere of $\mathrm{Al}$, resulting in stronger shielding and accordingly a lower chemical shift. To see only surface $\mathrm{Al}$ nuclei they used a surface sensitive $\quad\left\{{ }^{1} \mathrm{H}\right\} \quad{ }^{27} \mathrm{Al} \quad$ cross-polarization technique. Surface $\mathrm{Al}$ sites show a large variety of symmetry (tetrahedral, octahedral, trigonal bipyramid, square pyramid, trigonal), associated with very different EFG tensors. $\mathrm{OH}$ groups have a direct effect on the $\mathrm{Al}$ on which they are bound, changing not only the EFG but also the shielding (Wischert et al., 2014). The same computational approach would have been a good one to use for the exploration of isomorphously substituted effects on the hydrogen electric field gradient and nuclear shielding tensors in the Bronsted acid site of H-ZSM-5 zeolite. Instead the authors used an inadequate cluster model $\left(\mathrm{H}_{3} \mathrm{SiO}\right)_{3} \mathrm{Si}-\mathrm{O}(\mathrm{H})-\mathrm{X}\left(\mathrm{H}_{3} \mathrm{SiO}\right)_{3}$ where $\mathrm{X}=\mathrm{B}$, Al, Ga (Soltanali et al., 2014). 
A combined DNP surface enhanced NMRcomputational approach to the study of the active sites in $\mathrm{Sn}-\beta$ zeolite as a function of $\mathrm{Sn}$ loading has been reported (Wolf et al., 2014). This approach unambiguously shows the presence of framework $\mathrm{Sn}(\mathrm{IV})$-active sites in an octahedral environment, which probably correspond to so-called open and closed sites, respectively (i.e., Sn bound to 3 or 4 siloxy $\left(\mathrm{R}_{3} \mathrm{SiO}\right)$ groups of the zeolite framework). The authors used cluster models of increasing groups of atoms. For the most realistic cluster model, that includes the zeolite framework, the tetrahedral site has a calculated $\mathrm{Sn}$ isotropic chemical shift $\delta_{\text {iso }}=-396 \mathrm{ppm}$, in good agreement with experimental values and the chemical shift decreases by approximately $100 \mathrm{ppm}$ with the addition of each (1 or 2) coordinated water molecule. The calculated NMR parameters for the hexacoordinated Sn sites, resulting from the coordination/ reaction of at least two water molecules, are consistent with the experimental values. When Sn-sites have two water molecules in the first coordination sphere and one additional water molecule hydrogen-bonded to the coordinated $\mathrm{H}_{2} \mathrm{O}$, the calculated NMR parameters are in very good agreement with the experimental chemical shift parameters: $\delta_{\text {iso }}, \Omega$ and $\kappa$ values -654 ppm, 228 ppm, and 0.31 respectively for open sites and -684 ppm, 248 ppm, and 0.02 respectively for closed sites, allowing the assignment of the observed signals at $\delta=-654$ ppm to an open site and $\delta=-685 \mathrm{ppm}$ to a closed site, coordinated by two $\mathrm{H}_{2} \mathrm{O}$ molecules (Wolf et al., 2014). DFT GIPAW calculations using the CASTEP code predicted ${ }^{47 / 49} \mathrm{Ti}$ NMR parameters that may assist in spectral assignments and help correlate ${ }^{23} \mathrm{Na}$ and ${ }^{29} \mathrm{Si} \mathrm{NMR}$ resonances to crystallographic sites in 6 different titanosilicates ( $\mathrm{Xu}$ et al., 2014). In these systems, the ${ }^{23} \mathrm{Na}$ and ${ }^{39} \mathrm{~K}$ calculations probe the local environment of the counter cations.

Isotope Shifts. When protons from hydroxyl $(\mathrm{OH})$, sulfhydryl $(\mathrm{SH})$, and amino $\left(\mathrm{NH}_{2}\right)$ groups in a protein do not exchange readily with a deuterated solvent, resolved peaks are easily observed for ${ }^{13} \mathrm{C}$ nuclei that are one bond away from any one of these groups.
When the exchange rate is not too fast yet not slow enough to afford well separated peaks, a line shape analysis of the ${ }^{13} \mathrm{C}$ NMR signals of carbon nuclei near these polar groups, which are affected by deuterium-proton isotope shifts in a mixture of $\mathrm{H}_{2} \mathrm{O}$ and $\mathrm{D}_{2} \mathrm{O}$, can help identify such cases (Takeda et al., 2014). Deuterium induced isotope effects on ${ }^{6} \mathrm{Li}$ chemical shifts have been recently used to elucidate the structure of aggregates formed by butyllithium in diethyl ether in the presence of bromide ions (Böhler and Günther, 2015). In this particular case, the measured isotope shifts caused by deuterated butyllithium, the observed intensity pattern and multiplicity, and the shielding effect of a bromide ion are all taken into account to arrive at the identity of the nearest neighbors to lithium in the aggregate. Isotope effects through more than three bonds are expected to be sensitive to conformation. Böhm and coworkers have shown in the case of fluoroethane that a single D-substitution on C-2 can lead to isotope effects on ${ }^{19} \mathrm{~F}$ that vary depending on the conformation: $0.411 \mathrm{ppm}$ (anti) and 0.158 ppm (gauche). These numbers are obtained with $\operatorname{CCSD}(\mathrm{T}) / \mathrm{qz} 2 \mathrm{p}$ calculations, but the results from a lower level of theory, HF/tz2p, are not significantly different. Similar calculations performed on endo-2fluoronorbornanes suggest that the experimental assignments of conformers made previously are mistaken (Böhm et al., 2014). For the study of the tautomeric equilibria of pyridoyl benzoyl b-diketones, deuteriuminduced isotope effects on ${ }^{13} \mathrm{C}$ chemical shifts are found to corroborate conclusions derived from coupling constants regarding the equilibrium positions in these asymmetric diketones (Hansen et al., 2015). Two-bond deuterium isotope effects on ${ }^{1} \mathrm{H}$ NMR chemical shifts in $\mathrm{W}\left(\mathrm{PMe}_{3}\right)_{3} \mathrm{H}_{6-\mathrm{x}} \mathrm{D}_{\mathrm{x}}$ are of normal sign (the lighter isotopomer is deshielded) and are of similar magnitude to those observed in $\mathrm{CH}_{4-\mathrm{x}} \mathrm{D}_{\mathrm{x}}$, suggesting that these are indeed classical hydride compounds and not dihydrogen complexes (Sattler et al., 2014). The ${ }^{35 / 37} \mathrm{Cl}$ isotope effects on ${ }^{195} \mathrm{Pt}$ chemical shifts appear to be simpler in chlorohydroxidoplatinum(IV) complexes than in the corresponding aquachloro complexes. 
Engelbrecht and coworkers (Engelbrecht et al., 2015) have shown that for each ${ }^{195} \mathrm{Pt}$ NMR resonance of the $\left[\mathrm{Pt}^{35 / 37} \mathrm{Cl}_{6-\mathrm{n}}\left({ }^{16 / 18} \mathrm{OH}\right)_{\mathrm{n}}\right]^{2-}$, where $(\mathrm{n}=1-5)$, anions is resolved only into $[(6-n)+1$ for $n=1-5]$ peaks. Since calculations have not reached the desired accuracy for these systems, it is tentatively suggested that the greater trans influence of the hydroxido ligand is responsible for this difference. Lastly, one-bond oxygen isotope effects on ${ }^{99} \mathrm{Tc}$ chemical shifts in $\mathrm{TcO}_{4}{ }^{-}$have been recently reported, ${ }^{16 / 18} \Delta=-0.616 \mathrm{ppm}$ ${ }^{16 / 17} \Delta=-0.302$ ppm (Tarasov et al., 2015).

\section{Intermolecular Effects on Nuclear}

Shielding. An absolute shielding scale for the purpose of evaluating $a b$ initio methods is usually composed of resonance measurements in the gas phase extrapolated to zero density. In this manner, the experimental values correspond to those of an isolated molecule. There is no doubt that liquid to gas shifts are often significant. In order to incorporate medium effects on chemical shifts molecular dynamics simulations can be applied. Snapshots can be taken during the simulation and chemical shifts are then calculated from the clusters of molecules obtained during these snapshots. This approach has been performed on nitromethane. The calculated ${ }^{15} \mathrm{~N}$ chemical shift difference between liquid and gas phases is about $15 \mathrm{ppm}$. Compared to a dilute solution in cyclohexane, the ${ }^{15} \mathrm{~N}$ shielding in nitromethane in the gas phase is about 5 ppm more shielded (Gerber and Jolibois, 2015). Computational time and resources are not limitless so the size of the cluster employed in the calculation needs to be judiciously chosen. For instance, a number of acetonitrile solvent molecules in a $3.5 \AA$ thick shell, sampled from molecular dynamics simulation trajectories has been incorporated in the ${ }^{13} \mathrm{C}$ shielding computations of perylenic antenna molecules (Ozcan et al., 2014). Results show that for nuclei residing on the molecular perimeter acetonitrile causes deshielding while those near the center become shielded. Various approximations are also available where the solvent is modeled by a continuum. Albeit cheap, continuum models are generally not accurate enough to reproduce accurately solvent effects on shielding. It is therefore not surprising to see calculations that make use of such approximate methods not performing well in predicting chemical shifts due to chiral solutechiral solvent interactions (Kessler et al., 2015). For the solid phase, Holmes and coworkers have discovered what is necessary to perform shielding calculations using clusters of molecules that aim to reproduce shielding tensors (Holmes et al., 2014). The cluster must preserve the symmetry properties of the crystalline space group. Failing to observe symmetry leads to errors in the calculated principal shielding components.

Since intermolecular interactions affect what is observed in an NMR spectrum, NMR spectroscopy in the gas phase continues to be of great utility in host-guest systems. For instance, ${ }^{13} \mathrm{C}$ in $\mathrm{CO}_{2}$ has been exploited in probing cavities inside macrocyclic coordination supramolecules (Bassanetti et al., 2014). The residual anisotropy of the ${ }^{13} \mathrm{C}$ shielding tensor combined with pulse-field gradient experiments have been examined to study the diffusion of carbon dioxide along the channels of a metal-organic framework DMOF-1 ( $\mathrm{Zn}_{2}$ (benzene-(1,4)-dicarboxylate) ${ }_{2}$ (1,4-diazabicyclo [2.2.2]octane)) (Peksa et al., 2015). ${ }^{1} \mathrm{H}$ and ${ }^{13} \mathrm{C}$ NMR experiments have been applied to study the kinetics of methane replacement with carbon dioxide and nitrogen gas in methane gas hydrate prepared in porous silica gel matrices (Cha et al., 2015). Changes in ${ }^{3} \mathrm{He}$ shielding have been observed as a result of $\mathrm{He}$ gas interacting with sodium and calcium ions in the micropores of $5 \AA$ molecular sieve (Garbacz and Jackowski, 2015).

The position of protons in flexible hydroxyl groups is difficult to pinpoint by $\mathrm{x}$-ray diffraction. ${ }^{1} \mathrm{H}$ chemical shifts of the hydroxyl proton, of course, can tell whether a site is participating in hydrogen bonding or not. Oftentimes, ${ }^{1} \mathrm{H}$ chemical shifts are unable to provide specific conformational information not to mention that resolving these signals in the solid state are often very challenging. Filip and Filip (Filip and Filip, 2015) have 
suggested using ${ }^{13} \mathrm{C}$ chemical shifts instead since these are usually resolved and are quite sensitive to conformation. This approach has been demonstrated to be feasible in the case of quercetine dehydrate. Clusters containing an increasing number of pyrazole molecules have been utilized to determine the hydrogen bond effects on ${ }^{1} \mathrm{H}$ and ${ }^{15} \mathrm{~N}$ shieldings in crystalline pyrazole (Amini, 2014). By plotting the calculated shielding values against the reciprocal of the cluster size, values that correspond to the crystal limit can then be extrapolated.

Differences in the intermolecular packing of peptides are expected to manifest more in the chemical shifts of nuclei that are near the surface of the molecule. Using a model peptide (Ala-Gly) ${ }_{15}$, Asakura and coworkers have observed a distribution of ${ }^{1} \mathrm{H}$ NMR chemical shifts, suggesting that silk II form, the silk structure after spinning, is not a simple antiparallel $\beta$-sheet, but is heterogeneous due to different packing arrangements (Asakura et al., 2015). This is further supported by the dissimilar ${ }^{13} \mathrm{C}$ resonances observed for the side chain in alanine and the carbonyl carbons, as well as distinct amide ${ }^{15} \mathrm{~N}$ resonances, while seeing only small differences for $\mathrm{C} \alpha .{ }^{1} \mathrm{H}$ and ${ }^{19} \mathrm{~F}$ NMR chemical shifts combined with infrared data have led to the conclusion that there is no significant hydrogen bond interaction in 2-fluoroethanol (Cormanich et al., Seeming, 2014). The observed trend of ${ }^{19} \mathrm{~F}$ resonances becoming more shielded when a $\mathrm{F}$ atom participates in an attractive interaction and deshielded when the interaction is repulsive has been used to characterize the CF $\cdots$ FC interactions in selected organofluorine compounds (all-syn-1,2,3,4- and all-syn1,2,4,5-tetrafluorocyclohexane, 1,8-difluoronaphthalene, 4,5-difluorophenanthrene, and 2,2',5,5'-tetrafluorobiphenyl). In all cases, the CF $\cdots$ FC interactions are rendered repulsive (Cormanich et al., Analysis, 2014). Calculated ${ }^{13} \mathrm{C}$ and ${ }^{17} \mathrm{O}$ shielding tensors in carboxyl($\mathrm{COOH})$ and amide $\left(-\mathrm{CONH}_{2}\right)$ functionalized single-wall carbon nanotubes have been shown to correlate with hydrogen bond strength (Ghafouri et al., 2015). A range of about 40 ppm has been observed experimentally and reproduced theoretically for the isotropic shielding of ${ }^{17} \mathrm{O}$ in $\mathrm{H}_{2} \mathrm{O}$ bound in various hydrated crystalline amino acids (Michaelis et al., 2015). This variation in ${ }^{17} \mathrm{O}$ chemical shifts has been attributed to changes in $\mathrm{O}-\mathrm{H}$ bond distances and $\mathrm{H}-\mathrm{O}-\mathrm{H}$ bond angles. Hydrogen bond effects on ${ }^{13} \mathrm{C}$ shieldings have been examined in detail in 1,4-bis(4-cyanobenzyl)piperazine and 1,4bis(4-amidinobenzyl)piperazine tetrahydrochloride tetrahydrate (Rezler et al., 2014).

A relatively shielded resonance (-0.9 ppm from TMS) for methyl protons has been assigned to a methyl group lying closely to an aromatic group in a macrocyclic coordination supramolecule made from $\mathrm{Ag}^{+}$, thioether functionalized bis(pirazolyl)methane ligands, and $\mathrm{CF}_{3} \mathrm{SO}_{3}{ }^{-} / \mathrm{PF}_{6}{ }^{-}$as counter-anions (Bassanetti et al., 2014). Calculations involving dimers have been performed to deduce the structure of the dimer formed by a new shape-persistent macrocycle comprising two 2,3-triphenylene moieties bridged by m-phenylene ethynylenes (Chu et al., 2014). The geometry that produces closest agreement with experiment is one where the arenes are stacked, about $3.6 \AA$ apart, with a lateral displacement of about 3.5-4.0 $\AA$ along the molecular long axis. Computations of ${ }^{21} \mathrm{Ne}$ and ${ }^{39} \mathrm{Ar}$ shielding for rare gas atoms interacting with a furan suggest that these nuclei are more sensitive than ${ }^{3} \mathrm{He}$ to intermolecular interactions (Nieradka and Kupka, 2014).

Nucleus independent chemical shifts (NICS) have been applied in the evaluation of aromaticity in equatorial $\mathrm{BN}$-substituted nanocages (Anafcheh and Ghafouri, 2014). For fullerenes, BN substitution leads to less aromaticity while for nanocapsules, a greater aromaticity is suggested by calculated NICS. Current density calculations have shown that octaethylporphyrin zinc(II) dication, formally having $16 \pi$ electrons, is anti-aromatic (Fliegl et al., 2015). Spatial maps of magnetic properties have also been calculated and drawn for a variety of porphyrins, hemiporphyrazines and tetraoxo[8]circulenes 
(Kleinpeter et al., 2014), cyclopropane, the heteroanalogous oxirane, thiirane, and aziridine and various substituted mono-, bis-, and tris-cyclic analogues (Kleinpeter et al., Anisotropy, 2015), and a number of substituted para-nitro-pyridine $\mathrm{N}$-oxides (Kleinpeter et al, Interplay, 2015). These maps of NICS values around a functional group or aryl moiety are then employed to gauge the anisotropy and aromaticity effects arising from these moieties. Ring currents have been investigated using plane wave methods on semiconducting and carbon nanotubes (Ren et al., 2015). These calculations find that semiconducting nanotubes are more aromatic than metallic ones. Since deshielding of a proton is now considered as a defining evidence for hydrogen bonding, current density calculations have been recently performed to gain further insights on how hydrogen bonding affects chemical shifts. From these calculations, it is now evident that hydrogen bonding leads to a reduced electron density on the hydrogen causing a local reduction in the current density, which then manifests as a significant deshielding of the hydrogen nucleus (Monaco et al., 2015).

In a clever study modeling the NMR spectrum of ions diffusing in porous graphitic material, Grey et al. used NICS calculations to provide the shielding environment experienced by the ion at arbitrary positions in model slit pores of various widths to calculate the chemical shifts of the diffusing ion (Merlet et al., 2015). As diffusion inside pores is fast, the NMR spectrum of an ion trapped in a single mesopore will be a sharp peak with a poresize-dependent chemical shift. To account for the experimentally observed NMR line shapes, the simulations must model the relatively slow exchange between different pores. For this purpose the authors used a lattice gas model. The simplest lattice-gas models to simulate diffusion describe the diffusing species as non-interacting particles performing kinetic Monte Carlo moves on a lattice that contains both accessible sites (the fluid) and excluded sites (the porous matrix). They show that averaging over all possible diffusive trajectories of the ion can be achieved by a recursive numerical scheme called moment propagation, a very efficient method in which the computational effort scales as the number of lattice sites times the number of time steps.

The use of molecular clusters to take into account intermolecular effects is a relatively old method, many examples of which have been reviewed by us earlier (de Dios and Jameson, 2012). A recent example is the DFT calculation of ${ }^{13} \mathrm{C}$ shielding in polymorphs of sulfanilamide (Hartman and Beran, 2014). The authors refer to the method as "fragmentbased approach" and they treat the molecules beyond the one in the center as contributing higher order effects in a many-body expansion. In their present example, they report one-body and two-body calculations. Many methods of including long-range effects beyond the molecules of the cluster whose electrons are included in the quantum calculations had been employed. The simplest method is to use a continuum or reaction field model (e.g., PCM is very popular as an option in most electronic structure codes); others use electrostatic embedding such as partial charge field (de Dios et al., 1993), EIM (embedded ion method) (Stueber, 2006), the extended embedded ion method (EEIM) (Weber and Schmedt auf der Günne, 2010), or SCREEP (Surface Charge Representation of the Electrostatic Embedded Potential) (Stefanovich and Truong, 1998). For sulfanilamide the authors chose instead Stones' distributed multipole analysis (Stone, 1981; Stone and Alderton, 1985), which they then truncate at the leading term, that is, point charges, for this work. For three sulfanilamide polymorphs, they consider one-body, onebody charge-embedded, 2-body, 2-body charge-embedded models and compare their results with GIPAW calculations. The assignment of peaks to the correct polymorph is somewhat questionable at the 'one-body with charge embedding' level. The 'two-body with charge embedding' captures the trends in the ${ }^{13} \mathrm{C}$ chemical shifts much better and permits assigning the polymorphs. 
Calculations of NMR chemical shifts in proteins using fragments surrounded by appropriate models for the rest of the protein (charge fields, or molecular mechanics, or both) has been used for some time, since the pioneering work of de Dios et al. (de Dios et al., 1993). The fragment is typically treated at the highest correlated quantum mechanical level, the outer layers of the ONIOM (Morokuma's term) could be described using SCF and small basis set quantum calculations or else molecular mechanics, farther out, as needed, some electrostatic embedding may surround the first two layers, since carrying out self-consistent calculations in the presence of point charges is facile and cheap time-wise. Individual manual handling of the layers for preparing input to program suites still requires considerable attention, so an automated fragmentation $\mathrm{QM} / \mathrm{MM}$ approach is potentially very powerful (He et al., 2014). In this method, each amino acid and all the residues in its vicinity are automatically assigned as the QM region, using a distance cutoff for each residue-centric QM/MM calculation. They present a comparison of such calculations of ${ }^{1} \mathrm{H},{ }^{13} \mathrm{C}$ and ${ }^{15} \mathrm{~N}$ isotropic shielding for gas phase protein, protein in implicit solvent (a reaction field), and protein in explicit water molecules (including only the waters in the first and second solvation shell), which they compare against experimental data for chemical shifts in proteins GB3 and ubiquitin. The use of explicit solvent greatly improves the correlation plot against experiment. The easiest set to get excellent agreement with experiment is ${ }^{13} \mathrm{C}$ isotropic shifts. The effects of the electronic environment around the ${ }^{15} \mathrm{~N}$ nucleus is much more demanding to reproduce, as already shown in the early work (de Dios et al., 1993).

The ${ }^{129} \mathrm{Xe}$ nucleus has been used extensively in characterizing materials due to its great sensitivity to intermolecular interactions. Monte Carlo simulations have been incorporated in ${ }^{129} \mathrm{Xe}$ shielding calculations aimed at reproducing observed trends in ${ }^{129} \mathrm{Xe}$ chemical shifts as a liquid crystal undergoes a phase transition (Karjalainen et al., 2015). A combination of molecular dynamics and relativistic density functional calculations has likewise been carried out to reproduce the observed ${ }^{129}$ Xe NMR chemical shifts in room temperature ionic liquids (Saielli et al., Understanding, 2014). The results qualitatively agree with experiment as the calculated values suggest the same experimental trend, a greater shielding is observed when $\mathrm{PF}_{6}{ }^{-}$is the counterion as opposed to $\mathrm{Cl}^{-}$. The difference between these two ions leads to differences in both cage volume and the average charge surrounding the xenon nucleus, the two factors deemed important in these cases. This dependence of ${ }^{129} \mathrm{Xe}$ shielding on cage volume and charge is also evident in ${ }^{129} \mathrm{Xe}$ NMR spectra of Xe in clathrate hydrate lattices doped with $\mathrm{NH}_{4} \mathrm{~F}$ (Shin et al., 2014). ${ }^{129} \mathrm{Xe}$ NMR spectroscopy has proven to be useful as well in characterizing the anisotropic environment of the thermotropic nematic liquid crystal 4cyano-4'-pentylbiphenyl (Vemulapalli et al., 2015). On the other hand, ${ }^{131} \mathrm{Xe}$, a quadrupolar nucleus, via its EFG tensor, has been shown to report on the symmetry of phases in thermotropic nematic liquid crystals (Jokisaari and Zhu, 2014). Finally, Bryce and VigerGravel have recently authored an overview covering NMR experiments and interactions that are relevant to halogen bonding (Bryce and Viger-Gravel, 2015).

Absolute Shielding Scales. With new spinrotation data combined with high quality calculations of relativistic effects, absolute shielding scales for the ${ }^{17} \mathrm{O}$ and ${ }^{33} \mathrm{~S}$ nuclei have been recently refined (Komorovsky et al., 2015). The new values for ${ }^{17} \mathrm{O}$ are $328.4 \mathrm{ppm}$ for $\mathrm{H}_{2}{ }^{17} \mathrm{O}$, and $-59.1 \mathrm{ppm}$ for $\mathrm{C}^{17} \mathrm{O}$. For ${ }^{33} \mathrm{~S}$ in $\mathrm{H}_{2}{ }^{33} \mathrm{~S}$, the new value is $742.9 \mathrm{ppm}$. The relativistic effects for both water and carbon monoxide are quite small, 3.0 and $1.8 \mathrm{ppm}$, respectively, but for the heavier nucleus, ${ }^{33} \mathrm{~S}$, the corrections are quite substantial, $26 \mathrm{ppm}$. Spin-rotation data have also been obtained for various isotopomers of water yielding the following absolute shielding values for ${ }^{17} \mathrm{O}$ : $332.0 \mathrm{ppm} \mathrm{D}_{2}{ }^{17} \mathrm{O}$ and $329.6 \mathrm{ppm}$ for $\mathrm{HD}^{17} \mathrm{O}$ (Puzzarini et al., 2015). Both values contain relativistic corrections. 
${ }^{3} \mathrm{He},{ }^{129} \mathrm{Xe}$ and ${ }^{131} \mathrm{Xe}$ NMR measurements of resonance frequencies in the gas phase have been repeated to extract nuclear magnetic moments. The frequencies for ${ }^{129} \mathrm{Xe}$ and ${ }^{131} \mathrm{Xe}$ of an isolated Xe atom given as a fraction of the frequency of an isolated ${ }^{3} \mathrm{He}$ nucleus are 0.3630975 and 0.10763492 , respectively (Makulski, 2015). A similar procedure has been applied to ${ }^{83} \mathrm{Kr}$ which yields the following corresponding ratio, $v^{83} \mathrm{Kr} / \mathrm{v}^{3} \mathrm{He}=0.05051616$ (Makulski, 2014). These frequencies have also been converted to nuclear magnetic moments using previously calculated absolute shieldings that incorporate both relativistic and electron correlation effects.

\section{PERSPECTIVE}

Both plane wave and cluster calculations have extended shielding calculations to the condensed phase. Still, challenges remain in accurately incorporating dynamics into the computations. As one goes down the periodic table, relativistic effects as well as electron correlation and basis set size remain daunting. Nonetheless, there has been significant progress in this research field. Albeit accuracy in shielding computations still stands as a goal to be reached, how we understand the theoretical and physical aspects of nuclear magnetic shielding has greatly developed and matured. The study of nuclear shielding is definitely one area in which theoretical and experimental efforts have profoundly complemented each other. It is only expected that future experiments will continue to assist theoretical endeavors and computational work will remain indispensable as we explore ways to extract structural and dynamics information from NMR chemical shifts.

\section{ABBREVIATIONS}

B3LYP Becke 3-parameter Lee Yang Parr hybrid functional

CASSCF Complete active space SCF

CCSD Coupled-cluster singles and doubles
CCSD(T) CCSD model augmented by perturbative corrections for triple excitations

COSMO Conductor-like screening solvation model

CSGT Continuous set of gauge transformations

DFT Density functional theory

DHF Dirac Hartree-Fock

DNP Dynamic nuclear polarization

DOR Double rotation

EFG Electric field gradient

EEIM Extended embedded ion method

EIM Embedded ion method

EPR Electron paramagnetic resonance

FC Fermi contact

GIAO Gauge-including atomic orbitals

GIPAW Gauge-including projector augmented waves

HF Hartree-Fock

IGAIM Individual gauges for atoms in molecules

$\mathrm{KB}$

Kinetically balanced basis

KT2, KT3 Keal and Tozer functionals

LRESC Linear response elimination of small components

MAS Magic angle spinning

MP2 Second order Møller-Plesset

MQMAS Multiple quantum magic angle spinning 


\begin{tabular}{|c|c|}
\hline NBO & Natural bond orbital \\
\hline NICS & $\begin{array}{l}\text { Nucleus-independent chemical } \\
\text { shift }\end{array}$ \\
\hline NMR & Nuclear magnetic resonance \\
\hline ONIOM & $\begin{array}{l}\text { our own n-layered integrated } \\
\text { molecular orbital and } \\
\text { molecular mechanics method } \\
\text { (Morokuma) }\end{array}$ \\
\hline PBE & $\begin{array}{l}\text { Perdew-Burke-Ernzerhof } \\
\text { functional }\end{array}$ \\
\hline PCM & $\begin{array}{l}\text { Polarizable continuum solvent } \\
\text { model }\end{array}$ \\
\hline RAS & Restricted active space \\
\hline RLO & Rotational London orbitals \\
\hline RPA & $\begin{array}{l}\text { Random phase approximation } \\
\text { (first order polarization } \\
\text { propagator) }\end{array}$ \\
\hline SASS & $\begin{array}{l}\text { Switching angle sample } \\
\text { spinning }\end{array}$ \\
\hline SCF & Self-consistent field \\
\hline SCREEP & $\begin{array}{l}\text { Surface charge representation } \\
\text { of the electrostatic embedded } \\
\text { potential }\end{array}$ \\
\hline sf & Spin-free \\
\hline $\mathrm{SO}$ & Spin-orbit \\
\hline SOMO & $\begin{array}{l}\text { Singly occupied molecular } \\
\text { orbital }\end{array}$ \\
\hline SSNMR & Solid state NMR \\
\hline SWCNT & Single-walled carbon nanotube \\
\hline QED & Quantum electrodynamics \\
\hline $\mathrm{QM} / \mathrm{MM}$ & $\begin{array}{l}\text { Quantum mechanics } \\
\text { molecular mechanics approach }\end{array}$ \\
\hline VASP & $\begin{array}{l}\text { Vienna ab initio simulation } \\
\text { package }\end{array}$ \\
\hline
\end{tabular}

$\begin{array}{ll}\text { X2C } & \begin{array}{l}\text { Exact two-component } \\ \text { relativistic theory }\end{array} \\ \text { XC } & \text { Exchange correlation } \\ \text { ZFS } & \text { Zero-field splitting } \\ \text { ZORA } & \begin{array}{l}\text { Zeroth-order regular } \\ \text { approximation }\end{array}\end{array}$

\section{REFERENCES}

Aimola TJ, Lima DJP, Dias LC, Tormena CF, Ferreira MAB. ${ }^{1} \mathrm{H}$ chemical shift differences of Prelog-Djerassi lactone derivatives: DFT and NMR conformational studies. Org. Biomol. Chem. 2015; 13:2140-2145.

Alkan F, Dybowski C. Calculation of chemical-shift tensors of heavy nuclei: A DFT/ZORA investigation of ${ }^{199} \mathrm{Hg}$ chemicalshift tensors in solids, and the effects of cluster size and electronic-state approximations. Phys. Chem. Chem. Phys. 2014; 16:14298-14308.

Amini SK. A systematic investigation of cooperativity between two types of hydrogen bonding in the nonlinear clusters of an aromatic molecule: pyrazole. J. Mol. Struct. 2014; 1068:112-123.

Anafcheh M, Ghafouri R. BN belts: From small fullerenes to nanocapsules. J. Cluster Sci. 2014; 25:1173-1185.

Arshadi S, Bekhradnia AR, Mohammadi E, Asghari A. Disiline-doped boron nitride nanotubes: A computational study. J. Struct. Chem. 2014; 55:629-635

Asakura T, Ohata T, Kametani S, Okushita K, Yazawa K, Nishiyama Y, Nishimura K, Aoki A, Suzuki F, Kaji H, Ulrich AS, Williamson MP. Intermolecular packing in B. mori silk fibroin: Multinuclear NMR study of the model peptide (ala-gly)(15) defines a heterogeneous antiparallel antipolar mode of assembly in the silk II form. Macromolecules 2015; 48:28-36.

Autschbach J. Perspective: Relativistic effects. J. Chem. Phys. 2012; 136:150902. 
Bagno A, Saielli G. Addressing the stereochemistry of complex organic molecules by density functional theory-NMR. WIREsComput. Molec. Sci. 2015; 5:228-240.

Baias M, Lesage A, Aguado S, Canivet J, Moizan-Basle V, Audebrand N, Farrusseng D, Emsley L. Superstructure of a substituted zeolitic imidazolate metal-organic framework determined by combining proton solid-state NMR spectroscopy and DFT calculations. Angew. Chemie-Intl Ed. 2015; 54:5971-5976.

Bassanetti I, Comotti A, Sozzani P, Bracco S, Calestani G, Mezzadri F, Marchio L. Porous molecular crystals by macrocyclic coordination supramolecules. J. Am. Chem. Soc. 2014; 136:14883-14895.

Bevilaqua RCA, Rigo VA, Verissimo-Alves M, Miranda CR. NMR characterization of hydrocarbon adsorption on calcite surfaces: A first principles study. J. Chem. Phys. 2014; 141:204705.

Böhler B, Günther H. The aggregation behavior of butyllithium in diethyl ether in the presence of $\mathrm{LiBr}, \mathrm{LiClO}_{4}$, and phenyllithium: A deuterium-induced secondary ${ }^{6} \mathrm{Li}$ NMR isotope-effect study. Helv. Chim. Acta 2015; 98:427-446.

Böhm K, Banert K, Auer AA. Identifying stereoisomers by ab-initio calculation of secondary isotope shifts on NMR chemical shieldings. Molecules 2014; 19:5301-5312.

Boobalan MS, Ramalingam S, Amaladasan M, Tamilvendan D, Prabhu GV, Bououdina M. A computational perspective on equilibrium geometry, vibrational spectra and electronic structure of antioxidant active mannich base 1-[(pyridin-2-yl amino) methyl] pyrrolidine2,5-dione. J. Mol. Struct. 2014; 1072:153-172.

Bouty O, Delaye JM, Beuneu B, Charpentier T. Modelling borosilicate glasses of nuclear interest with the help of RMC, WAXS, neutron diffraction and ${ }^{11} \mathrm{~B}$ NMR. J. Non Cryst. Solids 2014; 401:27-31.
Bryce DL, Viger-Gravel J. Solid-state NMR study of halogen-bonded adducts. Top. Curr. Chem. 2015; 358:183-203.

Burgess KMN, Bryce DL. On the crystal structure of the vaterite polymorph of $\mathrm{CaCO}_{3}$ : A ${ }^{43} \mathrm{Ca}$ solid-state NMR and computational assessment. Solid State Nucl. Magn. Reson. 2015; 65:75-83.

Cadars S, Allix M, Brouwer DH, Shayib R, Suchomel M, Garaga MN, Rakhmatullin A, Burton AW, Zones SI, Massiot D, Chmelka BF. Long- and short-range constraints for the structure determination of layered silicates with stacking disorder.. Chem. Mater. 2014; 26:6994-7008.

Carnevale D, Ashbrook SE, Bodenhausen G. Solid-state NMR measurements and DFT calculations of the magnetic shielding tensors of protons of water trapped in barium chlorate monohydrate. RSC Advances 2014; 4:56248-56258.

Catalano J, Murphy A, Yao Y, Alkan F, Zurnbulyadis N, Centeno SA, Dybowski C. ${ }^{207} \mathrm{~Pb}$ and ${ }^{119} \mathrm{Sn}$ solid-state NMR and relativistic density functional theory studies of the historic pigment lead-tin yellow type I and its reactivity in oil paintings. J. Phys. Chem. A 2014; 118:7952-7958.

Catalano J, Murphy A, Yao Y, Yap GPA, Zumbulyadis N, Centeno SA, Dybowski C. Coordination geometry of lead carboxylates spectroscopic and crystallographic evidence. Dalton Trans. 2015; 44:2340-2347.

Cha M, Shin K, Lee H, Moudrakovski IL, Ripmeester JA, Seo Y. Kinetics of methane hydrate replacement with carbon dioxide and nitrogen gas mixture using in situ NMR spectroscopy. Environ. Sci. Technol. 2015; 49:1964-1971.

Cheng L, Gauss J, Stanton JF. Treatment of scalar-relativistic effects on nuclear magnetic shieldings using a spin-free exact-twocomponent approach. J. Chem. Phys. 2013; 139:054105. 
Chu M, Scioneaux AN, Hartley CS. Solutionphase dimerization of an oblong shapepersistent macrocycle. J. Org. Chem. 2014; 79:9009-9017.

Cormanich RA, Rittner R, Freitas MP, Bühl M. The seeming lack of CF...HO intramolecular hydrogen bonds in linear aliphatic fluoroalcohols in solution. Phys. Chem. Chem. Phys. 2014; 16:19212-19217.

Cormanich RA, Rittner R, O'Hagan D, Bühl M. Analysis of CF...FC interactions on cyclohexane and naphthalene frameworks. J. Phys. Chem. A 2014; 118:7901-7910.

Dawson DM, Ashbrook SE. Investigating relationships between the crystal structure and ${ }^{31} \mathrm{P}$ isotropic chemical shifts in calcined aluminophosphates. J. Phys. Chem. C 2014; 118:23285-23296.

de Dios AC, Jameson CJ. Recent advances in nuclear shielding calculations. In Annual Reports on NMR Spectroscopy, Webb GA, editor. London: Elsevier Ltd; 2012. Vol. 77. p. $1-80$.

de Dios AC, Pearson JG, Oldfield E. Secondary and tertiary structural effects on protein NMR chemical shifts: an ab initio approach. Science 1993; 260:1491-1496.

Dervisoglu R, Middlemiss DS, Blanc F, Lee Y, Morgan D, Grey CP. Joint experimental and computational ${ }^{17} \mathrm{O}$ and ${ }^{1} \mathrm{H}$ solid state NMR study of $\mathrm{Ba}_{2} \mathrm{In}_{2} \mathrm{O}_{4}(\mathrm{OH})_{2}$ structure and dynamics. Chem. Mater. 2015; 27:3861-3873.

Diehl BG, Watts HD, Kubicki JD, Regner MR, Ralph J, Brown NR. Towards ligninprotein crosslinking: Amino acid adducts of a lignin model quinone methide. Cellulose 2014; 21:1395-1407.

Engelbrecht L, Murray P, Koch KR. Isotope effects in ${ }^{195} \mathrm{Pt}$ NMR spectroscopy: Unique ${ }^{35 / 37} \mathrm{Cl}^{-}$and ${ }^{16 / 18} \mathrm{O}$-resolved "fingerprints" for all $\left[\mathrm{PtCl}_{6-\mathrm{n}}(\mathrm{OH})_{\mathrm{n}}\right]^{2-} \quad(\mathrm{n}=1-5)$ anions in an alkaline solution and the implications of the trans influence. Inorg. Chem. 2015; 54:27522764.
Fedorov SV, Rusakov YY, Krivdin LB. Towards the versatile DFT and MP2 computational schemes for ${ }^{31} \mathrm{P}$ NMR chemical shifts taking into account relativistic corrections. Magn. Reson. Chem. 2014; 52:699-710.

Filip X, Filip C. Can the conformation of flexible hydroxyl groups be constrained by simple NMR crystallography approaches? The case of the quercetin solid forms. Solid State Nucl. Magn. Reson. 2015; 65:21-28.

Fliegl H, Pichierri F, Sundholm D. Antiaromatic character of 16 pi electron octaethylporphyrins: Magnetically induced ring currents from DFT-GIMIC calculations. J. Phys. Chem. A 2015; 119:2344-2350.

Flygare WH. Spin-Rotation interaction and magnetic shielding in molecules. J. Chem. Phys. 1964; 41:793-800.

Gambuzzi E, Charpentier T, Menziani MC, Pedone A. Computational interpretation of ${ }^{23} \mathrm{Na}$ MQMAS NMR spectra: A comprehensive investigation of the $\mathrm{Na}$ environment in silicate glasses. Chem. Phys. Lett. 2014; 612:56-61.

Garay PG, Martin OA, Scheraga HA, Vila JA. Factors affecting the computation of the ${ }^{13} \mathrm{C}$ shielding in disaccharides. J. Comput. Chem. 2014; 35:1854-1864.

Garbacz P, Jackowski K. NMR shielding of ${ }^{3} \mathrm{He}$ in the micropores of zeolites. Micro. Meso. Mater. 2015; 205:52-55.

Gendron F, Sharkas K, Autschbach J. Calculating NMR chemical shifts for paramagnetic metal complexes from firstprinciples. J. Phys. Chem. Lett. 2015; 6:21832188.

Gerber IC, Jolibois F. Theoretical gas to liquid shift of ${ }^{15} \mathrm{~N}$ isotropic nuclear magnetic shielding in nitromethane using ab initio molecular dynamics and GIAO/GIPAW calculations. Phys. Chem. Chem. Phys. 2015; 17:12222-12227. 
Ghafouri R, Ektefa F, Zahedi M. Characterization of hydrogen bonds in the end-functionalized single-wall carbon nanotubes: A DFT study. Nano 2015; 10:1550036.

Guo C, Hou G, Lu X, O'Hare B, Struppe J, Polenova T. Fast magic angle spinning NMR with heteronucleus detection for resonance assignments and structural characterization of fully protonated proteins. J. Biomol. NMR 2014; 60:219-229.

Hansen PE, Borisov EV, Lindon JC. Determination of the tautomeric equilibria of pyridoyl benzoyl beta-diketones in the liquid and solid state through the use of deuterium isotope effects on ${ }^{1} \mathrm{H}$ and ${ }^{13} \mathrm{C}$ NMR chemical shifts and spin coupling constants. Spectrochim. Acta A 2015; 136:107-112.

Hartman JD, Beran GJO. Fragment-based electronic structure approach for computing nuclear magnetic resonance chemical shifts in molecular crystals. J. Chem. Theor. Comput. 2014; 10:4862-4872.

He X, Zhu T, Wang X, Liu J, Zhang JZH. Fragment quantum mechanical calculation of proteins and its applications. Acc. Chem. Res. 2014; 47:2748-2757.

Hill DE, Vasdev N, Holland JP. Evaluating the accuracy of density functional theory for calculating ${ }^{1} \mathrm{H}$ and ${ }^{13} \mathrm{C}$ NMR chemical shifts in drug molecules. Comput. Theor. Chem. 2015; 1051:161-172.

Holmes ST, Iuliucci RJ, Mueller KT, Dybowski C . Density functional investigation of intermolecular effects on ${ }^{13} \mathrm{C}$ NMR chemical-shielding tensors modeled with molecular clusters. J. Chem. Phys. 2014; 141:164121.

Jameson CJ, de Dios AC. Theoretical and physical aspects of nuclear shielding. In Nuclear Magnetic Resonance, KamienskaTrela K, editor. London: Royal Society of Chemistry; 2013. Vol. 42. p. 45-77.
Jameson CJ, de Dios AC. Theoretical and physical aspects of nuclear shielding. In Nuclear Magnetic Resonance, KamienskaTrela K, editor. London: Royal Society of Chemistry; 2014. Vol. 43. p. 49-80.

Jameson CJ, de Dios AC. Theoretical and physical aspects of nuclear shielding. In Nuclear Magnetic Resonance, KamienskaTrela K, editor. London: Royal Society of Chemistry; 2015. Vol. 44. p. 46-75.

Jankowska M, Kupka T, Stobinski L, Kaminsky J. DFT studies on armchair $(5,5)$ SWCNT functionalization. modification of selected structural and spectroscopic parameters upon two-atom molecule attachment. J. Molec. Graph. Model. 2015; 55:105-114.

Jaszunski M, Demissie TB, Ruud K. Spinrotation and NMR shielding constants in XF molecules (X = B, Al, Ga, In, and Tl). J. Phys. Chem. A 2014; 118:9588-9595.

Jokisaari J, Zhu J. Xenon NMR of phase biaxiality in liquid crystals. Magn. Reson. Chem. 2014; 52:556-559.

Kamihara T, Mizuno T, Shoji A, Takegoshi K. Conformational characterization of lefthanded helices in poly(beta-benzyl Laspartate) by ${ }^{13} \mathrm{C}$ chemical shift anisotropy using solid-state NMR. Macromolecules 2015; 48:629-636.

Karhu AJ, Pakkanen OJ, Rautiainen JM, Oilunkaniemi R, Chivers T, Laitinen RS. Experimental and computational ${ }^{77} \mathrm{Se}$ NMR investigations of the cyclic eight-membered selenium imides $1,3,5,7-\mathrm{Se}_{4}(\mathrm{NR})_{4}(\mathrm{R}=\mathrm{Me}$, $\left.{ }^{\mathrm{t}} \mathrm{Bu}\right)$ and 1,5-Se ${ }_{6}(\mathrm{NMe})_{2}$. Inorg. Chem. 2015; 54:4990-4997.

Karjalainen J, Vaara J, Straka M, Lantto P. Xenon NMR of liquid crystals confined to cylindrical nanocavities: A simulation study. Phys. Chem. Chem. Phys. 2015; 17:7158-7171. 
Keal TW, Tozer DJ. The exchange-correlation potential in Kohn-Sham nuclear magnetic resonance shielding calculations. J. Chem. Phys., 2003, 119, 3015-3024.

Keal TW, Tozer DJ. A semiempirical generalized gradient approximation exchangecorrelation functional. J. Chem. Phys. 2004; 121:5654-5660.

Kessler J, Dracinsky M, Bour P. Determination of absolute configuration in chiral solvents with nuclear magnetic resonance. A combined molecular Dynamics/Quantum chemical study. J. Phys. Chem. A 2015; 119:5260-5268.

Kim G, Griffin JM, Blanc F, Haile SM, Grey $\mathrm{CP}$. Characterization of the dynamics in the protonic conductor $\mathrm{CsH}_{2} \mathrm{PO}_{4}$ by ${ }^{17} \mathrm{O}$ solidstate NMR spectroscopy and first-principles calculations: Correlating phosphate and protonic motion. J. Am. Chem. Soc. 2015; 137:3867-3876.

Kleinpeter E, Koch A, Schulz S, Wacker P. Interplay of para- and diatropic ring currents [(anti)aromaticity] of macrocyclic rings subject to conformational influences, further annelation and hydrogenation of aromatic ring moieties. Tetrahedron 2014; 70:9230-9239.

Kleinpeter E, Kriiger S, Koch A. Anisotropy effect of three-membered rings in ${ }^{1} \mathrm{H}$ NMR spectra: Quantification by TSNMRS and assignment of the stereochemistry. J. Phys. Chem. A 2015; 119:4268-4276.

Kleinpeter E, Michaelis M, Koch A. Are paranitro-pyridine $\mathrm{N}$-oxides quinonoid or benzenoid? an answer given by spatial NICS (TSNMRS). Tetrahedron 2015; 71:2273-2279.

Kobayashi T, Gupta S, Caporini MA, Pecharsky VK, Pruski M. Mechanism of solidstate thermolysis of ammonia borane: $\mathrm{A}{ }^{15} \mathrm{~N}$ NMR study using fast magic-angle spinning and dynamic nuclear polarization. J. Phys. Chem. C 2014; 118:19548-19555.
Komasa A, Katrusiak A, Kazmierczak M, Dega-Szafran Z, Szafran M. Spectroscopic, structural and theoretical investigation of bis(4-trimethylammoniumbenzoate)

hydroiodide hydrate. Spectrochim. Acta A 2015; 136:1149-1156.

Komorovsky S, Repisky M, Malkin E, Ruud K, Gauss J. Communication: The absolute shielding scales of oxygen and sulfur revisited. J. Chem. Phys. 2015; 142:091102.

Kong X, Tang A, Wang R, Ye E, Terskikh V, Wu G. Are the amide bonds in $\mathrm{N}$-acyl imidazoles twisted? A combined solid-state ${ }^{17} \mathrm{O}$ NMR, crystallographic, and computational study. Can. J. Chem. 2015; 93:451-458.

Kubica D, Gryff-Keller A. Orotic acid in water solution, a DFT and ${ }^{13} \mathrm{C}$ NMR spectroscopic study. J. Phys. Chem. B 2015; 119:5832-5838.

Kusumi R, Kimura F, Kimura T. Determination of ${ }^{31} \mathrm{P}$ chemical shift tensor from microcrystalline powder by using a magnetically oriented microcrystal array. Crystal Growth \& Design 2015; 15:718-722.

Lazzeretti P. Invariance of molecular response properties under a coordinate translation. Intl. J. Quantum Chem. 2014; 114:1364-1392.

Leniak A, Jazwinski J. DFT calculations of ${ }^{15} \mathrm{~N}$ NMR shielding constants, chemical shifts and complexation shifts in complexes of rhodium(II) tetraformate with some nitrogenous organic ligands. J. Mol. Struct. 2015; 1083:336-342.

Li M, Yehl J, Hou G, Chatterjee PB, Goldbourt A, Crans DC, Polenova T. NMR crystallography for structural characterization of oxovanadium $(\mathrm{V})$ complexes: Deriving coordination geometry and detecting weakly coordinated ligands at atomic resolution in the solid state. Inorg. Chem. 2015; 54:1363-1374.

Li Z, Xiao Y, Liu W. On the spin separation of algebraic two-component relativistic Hamiltonians. J. Chem. Phys. 2012; 137:154114. 
Li Z, Xiao Y, Liu W. On the spin separation of algebraic two-component relativistic Hamiltonians: Molecular properties. J. Chem. Phys. 2014; 141:054111.

Liu W. Advances in relativistic molecular quantum mechanics. Phys. Rep. 2014; 537:5989.

Liu W. Perspective: Relativistic Hamiltonians. Int. J. Quantum Chem. 2014; 114:983-986.

Liu W. Effective Quantum Electrodynamics Hamiltonians, a Tutorial Review. Int. J. Quantum Chem. 2015; 115:6310640.

Liu Y, Junk T, Liu Y, Tzeng N, Perkins R. Benchmarking quantum mechanical calculations with experimental NMR chemical shifts of 2-HADNT. J. Mol. Struct. 2015; 1086:43-48.

Lynch BJ, Fast PL, Harris M, Truhlar DG. Adiabatic connection for kinetics. J. Phys. Chem. A 2000; 104:4811-4815.

Makulski W. ${ }^{129} \mathrm{Xe}$ and ${ }^{131} \mathrm{Xe}$ nuclear magnetic dipole moments from gas phase NMR spectra. Magn. Reson. Chem. 2015; 53:273279.

Makulski W. ${ }^{83} \mathrm{Kr}$ nuclear magnetic moment in terms of that of ${ }^{3} \mathrm{He}$. Magn. Reson. Chem. 2014; 52:430-434.

Maldonado AF, Aucar GA. Relativistic and electron-correlation effects on the nuclear magnetic resonance shieldings of molecules containing tin and lead atoms. J. Phys. Chem. A 2014; 118:7863-7875.

Maldonado AF, Aucar GA, Melo JI . Coredependent and ligand-dependent relativistic corrections to the nuclear magnetic shieldings in $\mathrm{MH}_{4-\mathrm{n}} \mathrm{Y}_{\mathrm{n}}(\mathrm{n}=0-4 ; \mathrm{M}=\mathrm{Si}, \mathrm{Ge}, \mathrm{Sn}$, and $\mathrm{Y}=$ H, F, Cl, Br, I) model compounds. J. Molec. Model. 2014; 20:2417.

Martin B, Autschbach J. Temperature dependence of contact and dipolar NMR chemical shifts in paramagnetic molecules. J. Chem. Phys. 2015; 142:054108.
Merlet C, Forse AC, Griffin JM, Frenkel D, Grey CP. Lattice simulation method to model diffusion and NMR spectra in porous materials. J. Chem. Phys, 2015; 142:094701.

Michaelis VK, Keeler EG, Ong T, Craigen KN, Penzel S, Wren JEC, Kroeker S, Griffin RG. Structural insights into bound water in crystalline amino acids: Experimental and theoretical ${ }^{17}$ O NMR. J. Phys. Chem. B 2015; 119:8024-8036.

Monaco G, Della Porta P, Jablonski M, Zanasi R. Topology of the magnetically induced current density and proton magnetic shielding in hydrogen bonded systems. Phys. Chem. Chem. Phys. 2015; 17:5966-5972.

Monserrat B, Needs RJ, Pickard CJ. Temperature effects in first-principles solid state calculations of the chemical shielding tensor made simple. J. Chem. Phys. 2014; 141:134113.

Munoz-Castro A. Axis-dependent magnetic behavior of $\mathrm{C}_{60}$ and $\mathrm{C}_{60}{ }^{10+}$. consequences of spherical aromatic character. Chem. Commun. 2015; 51:10287-10290.

Nieradka M, Kupka T. Sensitivity of noble gas NMR parameters to the heterocyclic ring proximity. density functional theory studies of Ne-furan and Ar-furan complexes. Chem. Heterocyc. Compounds 2014; 50:429-437.

Nimmo II JP, Kroll P. First-principles calculations and analysis of ${ }^{29} \mathrm{Si}$ nuclear magnetic resonance chemical shifts in silicon oxycarbide ceramics. J. Phys. Chem. C 2014; 118:29952-29961.

Nozirov F, Kupka T, Stachow M. Theoretical prediction of structural, vibrational and NMR parameters of plastic optical fiber (POF) material precursors. Cis and trans perhydroand perfluoro-2-methylene-4,5-dimethyl-1,3dioxolanes. J. Molec. Graph. Model. 2014; 52:36-45. 
O'Keefe CA, Johnston KE, Sutter K, Autschbach J, Gauvin R, Trebosc J, Delevoye L, Popoff N, Taoufik M, Oudatchin K, Schurko W. An investigation of chlorine ligands in transition-metal complexes via ${ }^{35} \mathrm{Cl}$ solid-state NMR and density functional theory calculations. Inorg. Chem. 2014; 53:95819597.

Oliveira LBA, Colherinhas G, Fonseca TL, Castro MA. Spectroscopic properties of vitamin $\mathrm{E}$ models in solution. Chem. Phys. Lett. 2015; 628:49-53.

Ozcan N, Mares J, Sundholm D, Vaara J. Characteristic spectral patterns in the ${ }^{13} \mathrm{C}$ nuclear magnetic resonance spectra of hexagonal and crenellated graphene fragments. Phys. Chem. Chem. Phys. 2014; 16:22309-22320.

Paluch P, Pawlak T, Oszajca M, Lasocha W, Potrzebowski MJ. Fine refinement of solid state structure of racemic form of phosphotyrosine employing NMR crystallography approach. Solid State Nucl. Magn. Reson. 2015; 65:2-11.

Pandey MK, Malon M, Ramamoorthy A, Nishiyama Y. Composite-180 degrees pulsebased symmetry sequences to recouple proton chemical shift anisotropy tensors under ultrafast MAS solid-state NMR spectroscopy. J. Magn. Reson. 2015; 250:45-54.

Peksa M, Lang J, Stallmach F. ${ }^{13} \mathrm{C}$ NMR study of diffusion anisotropy of carbon dioxide adsorbed in nanoporous DMOF-1. Micro. Meso. Mater. 2015; 205:11-15.

Pelloni S, Monaco G, Della Porta P, Zanasi R, Lazzeretti P. Delocalized currents without a ring of bonded atoms: Strong delocalized electron currents induced by magnetic fields in noncyclic molecules. J. Phys. Chem. A 2014; 118:3367-3375.

Pennanen TO, Vaara J. Nuclear magnetic resonance chemical shift in an arbitrary electronic spin state. Phys. Rev. Lett. 2008; 100:133002.
Pichumani K, George G, Hebbar S, Chatterjee B, Raghothama S. Effects of hydrogen bonding on amide-proton chemical shift anisotropy in a proline-containing model peptide. Chem. Phys. Lett. 2015; 627:126-129.

Pindelska E, Sokal A, Szeleszczuk L, Pisklak DM, Kolodziejski W. Solid-state NMR studies of theophylline co-crystals with dicarboxylic acids. J. Pharm. Biomed. Anal. 2014; 100:322328.

Pindelska E, Szeleszczuk L, Pisklak DM, Mazurek A, Kolodziejski W. Solid-state NMR as an effective method of polymorphic analysis: Solid dosage forms of clopidogrel hydrogensulfate. J. Pharm. Sci. 2015; 104:106113.

Presti D, Pedone A, Menziani MC. Unraveling the polymorphism of $[(p$-cymene $) \mathrm{Ru}(\kappa \mathrm{N}$ INA) $\mathrm{Cl}_{2}$ ] through dispersion-corrected DFT and NMR GIPAW calculations. Inorg. Chem. 2014; 53:7926-7935.

Puzzarini C, Cazolli G, Harding ME, Vazquez $\mathrm{J}$, Gauss J. The hyperfine structure in the rotational spectra of $\mathrm{D}_{2}{ }^{17} \mathrm{O}$ and $\mathrm{HD}^{17} \mathrm{O}$ : Confirmation of the absolute nuclear magnetic shielding scale for oxygen, J. Chem. Phys. 2015; 142:124308

Ramsey NF. Magnetic shielding of nuclei in molecules. Phys. Rev. 1950; 78:699-703.

Reddy GNM, Cook DS, Iuga D, Walton RI, Marsh A, Brown SP. An NMR crystallography study of the hemihydrate of 2 ', 3 '-Oisopropylidineguanosine. Solid State Nucl. Magn. Reson. 2015; 65:41-48.

Ren J, Eckert H. Intermediate role of gallium in oxidic glasses: Solid state NMR structural studies of the $\mathrm{Ga}_{2} \mathrm{O}_{3}-\mathrm{NaPO}_{3}$ system. J. Phys. Chem. C 2014; 118:15386-15403.

Ren P, Zheng A, Xiao J, Pan X, Bao X. Exploring the ring current of carbon nanotubes by first-principles calculations. Chem. Sci. 2015; 6:902-908. 
Reynolds RD, Shiozaki T. Fully relativistic self-consistent field under a magnetic field. Phys. Chem. Chem. Phys. 2015; 17:1428014283.

Rezler M, Zolek T, Wolska I, Maciejewska D. Structural aspects of intermolecular interactions in the solid state of 1,4dibenzylpiperazines bearing nitrile or amidine groups. Acta Crystallog. B 2014; 70:820-827.

Rouf SA, Mares J, Vaara J. ${ }^{1} \mathrm{H}$ chemical shifts in paramagnetic $\mathrm{Co}(\mathrm{II})$ pyrazolylborate complexes: A first-principles study. J. Chem. Theor. Comput. 2015; 11:1683-1691.

Saielli G, Bini R, Bagno A. Computational ${ }^{19} \mathrm{~F}$ NMR. 2. Organic compounds. RSC Advances 2014; 4:41605-41611.

Saielli G, Bagno A, Castiglione F, Simonutti R, Mauri M, Mele A. Understanding cage effects in imidazolium ionic liquids by ${ }^{129} \mathrm{Xe}$ NMR: MD simulations and relativistic DFT calculations. J. Phys. Chem. B 2014; 118:13963-13968.

Samultsev DO, Semenov VA, Krivdin LB. On the accuracy of the GIAO-DFT calculation of ${ }^{15} \mathrm{~N}$ NMR chemical shifts of the nitrogencontaining heterocycles - a gateway to better agreement with experiment at lower computational cost. Magn. Reson. Chem. 2014; 52:222-230.

Sas EB, Kurt M, Karabacak M, Poiyamozhi A, Sundaraganesan N. FT-IR, FT-raman, dispersive raman, NMR spectroscopic studies and $\mathrm{NBO}$ analysis of 2-bromo-1Hbenzimidazol by density functional method. J. Mol. Struct. 2015; 1081:506-518.

Sattler A, Zuzek AA, Parkin G. Molecular structure of $\mathrm{W}\left(\mathrm{PMe}_{3}\right)_{3} \mathrm{H}_{6}$ in the solid state and in solution. Inorg. Chim. Acta, 2014, 422, 102 108.

Semenov VA, Samultsev DO, Krivdin LB. Solvent effects in the GIAO-DFT calculations of the ${ }^{15} \mathrm{~N}$ NMR chemical shifts of azoles and azines. Magn. Reson. Chem. 2014; 52:686-693.
Seymour VR, Eschenroeder ECV, Wright PA, Ashbrook SE. An NMR crystallographic approach to monitoring cation substitution in the aluminophosphate STA-2. Solid State Nucl. Magn. Reson. 2015; 65:64-74.

Shin K, Moudrakovski IL, Davari MD, Alavi S, Ratcliffe CI, Ripmeester JA. Crystal engineering the clathrate hydrate lattice with $\mathrm{NH}_{4}$ F. Crystengcomm 2014; 16:7209-7217.

Soltanali S, Halladj R, Ektefa F. A computational exploration into isomorphously substituted effects on hydrogen electric field gradient and chemical shielding tensors in the H-ZSM-5 zeolite. Asia-Pacific J. Chem. Eng. 2014; 9:574-580.

Soncini A, Van den Heuvel W. Communication: Paramagnetic NMR chemical shift in a spin state subject to zerofield splitting. J. Chem. Phys. 2013; 138:021103.

Stefanovich EV, Truong TN. A simple method for incorporating Madelung field effects into $a b$ initio embedded cluster calculations of crystals and macromolecules. J. Phys. Chem. B 1998; 102:3018-3022.

Stueber D. The embedded ion method: A new approach to the electrostatic description of crystal lattice effects in chemical shielding calculations. Concepts Magn. Reson. A 2006; 28:347-368.

Stone AJ. Distributed multipole analysis, or how to describe a molecular charge distribution . Chem. Phys. Lett. 1981; 83:233239.

Stone AJ, Alderton M. Distributed multipole analysis. Methods and applications. Mol. Phys. 1985; 56:1047-1064.

Struppe J, Zhang Y, Rozovsky S. ${ }^{77}$ Se chemical shift tensor of L-selenocystine: Experimental NMR measurements and quantum chemical investigations of structural effects. J. Phys. Chem. B 2015; 119: 36433650 . 
Sun Q, Xiao Y, Liu W. Exact two-component relativistic theory for NMR parameters: General formulation and pilot application. J. Chem. Phys. 2012; 137:174105.

Sykina K, Bureau B, Le Polles L, Roiland C, Deschamps M, Pickard CJ, Furet E. A combined ${ }^{77} \mathrm{Se}$ NMR and molecular dynamics contribution to the structural understanding of the chalcogenide glasses. Phys. Chem. Chem. Phys. 2014; 16:17975-17982.

Szafran M, Komasa A, Ostrowska K, Katrusiak A, Dega-Szafran Z. Spectral and structural studies of dimethylphenyl betaine hydrate. Spectrochim. Acta A 2015; 136:12161226.

Takeda M, Miyanoiri Y, Terauchi T, Yang C, Kainosho M. Use of H/D isotope effects to gather information about hydrogen bonding and hydrogen exchange rates. J. Magn. Reson. 2014; 241:148-154.

Tarasov VP, Kirakosyan GA, German KE. Oxygen isotope effect on NMR parameters of pertechnetate anion $\mathrm{TcO}_{4}^{-}$. Russ. J. Phys. Chem. B 2015; 9:185-192.

Teale AM, Lutnas OB, Helgaker T, Tozer DJ, and Gauss J. Benchmarking density-functional theory calculations of NMR shielding constants and spin-rotation constants using accurate coupled-cluster calculations. J. Chem. Phys. 2013; 138:024111.

Tian Y, Lu GJ, Marassi FM, Opella SJ. Structure of the membrane protein MerF, a bacterial mercury transporter, improved by the inclusion of chemical shift anisotropy constraints. J. Biomol. NMR 2014; 60:67-71.

Van den Heuvel W, Soncini A. NMR chemical shift as analytical derivative of the Helmholtz free energy. J. Chem. Phys. 2013; 138:054113.

Vemulapalli PBS, Police N, Bharatam J. ${ }^{129} \mathrm{Xe}$ NMR investigation of the anisotropic environment of a thermotropic nematic liquid crystal 4-cyano-4 '-pentylbiphenyl. Molec. Cryst. Liq. Cryst. 2015; 607:70-77.
Vicha J, Foroutan-Nejad C, Pawlak T, Munzarova ML, Straka M, Marek R. Understanding the electronic factors responsible for ligand spin-orbit NMR shielding in transition-metal complexes. J. Chem. Theor. Comput. 2015; 11:1509-1517.

Viesser RV, Ducati LC, Autschbach J, Tormena CF. Effects of stereoelectronic interactions on the relativistic spin-orbit and paramagnetic components of the ${ }^{13} \mathrm{C}$ NMR shielding tensors of dihaloethenes. Phys. Chem. Chem. Phys. 2015; 17:19315-19324 .

Vogt FG, Williams GR, Strohmeier M, Johnson MN, Copley RCB. Solid-state NMR analysis of a complex crystalline phase of ronacaleret hydrochloride. J. Phys. Chem. B 2014; 118:10266-10284 .

Walder BJ, Dey KK, Davis MC, Baltisberger JH, Grandinetti PJ. Two-dimensional NMR measurement and point dipole model prediction of paramagnetic shift tensors in solids. J. Chem. Phys. 2015; 142:014201.

Weber J, Schmedt auf der Günne J. Calculation of NMR parameters in ionic solids by an improved self-consistent embedded cluster method. Phys. Chem. Chem. Phys. 2010; 12:583-603.

Widdifield CM, Perras FA, Bryce DL. Solidstate ${ }^{185 / 187}$ Re NMR and GIPAW DFT study of perrhenates and $\operatorname{Re}_{2}(C O)_{10}$ : Chemical shift anisotropy, NMR crystallography, and a metal-metal bond. Phys. Chem. Chem. Phys. 2015; 17:10118-10134.

Wischert R, Florian P, Coperet C, Massiot D, Sautet P. Visibility of al surface sites of gamma-alumina: A combined computational and experimental point of view. J. Phys. Chem. C 2014; 118:15292-15299.

Wolf P, Valla M, Rossini AJ, Comas-Vives A, Nunez-Zarur F, Malaman B, Lesage A, Emsley L, Coperet C, Hermans I. NMR signatures of the active sites in Sn $\beta$ zeolite. Angew. Chemie-Intl Ed. 2014; 53:1017910183. 
Xiao Y, Sun Q, Liu W. Fully relativistic theories and methods for NMR parameters .Theor. Chem. Acc. 2012; 131:1080.

Xiao Y, Zhang, Liu W. Relativistic Theory of nuclear spin-rotation tensor with kinetically balanced rotational London orbitals, J. Chem. Phys. 2014; 141:164110.

$\mathrm{Xu} J$, Lucier BEG, Lin Z, Sutrisno A, Terskikh VV, Huang Y. New insights into the short-range structures of microporous titanosilicates as revealed by ${ }^{47 / 49} \mathrm{Ti},{ }^{23} \mathrm{Na},{ }^{39} \mathrm{~K}$, and ${ }^{29} \mathrm{Si}$ solid-state NMR spectroscopy. J. Phys. Chem. C 2014; 118:27353-27365.
Yan S, Yao L. DFT application in conformational determination of cellobiose. Carbohydr. Res. 2015; 404:117-123.

Yoshizawa T, Hada M. Gauge-origin dependence of NMR shielding constants in the Douglas-Kroll-Hess method. Chem. Phys. Lett. 2015; 618:132-141.

Zhang C, Patschinski P, Stephenson DS, Panisch R, Wender JH, Holthausen MC, Zipse H. The calculation of ${ }^{29} \mathrm{Si}$ NMR chemical shifts of tetracoordinated silicon compounds in the gas phase and in solution. Phys. Chem. Chem. Phys. 2014; 16:1664216650. 